\title{
Structural and Metabolic Biomarkers in Multiple Sclerosis
}

\section{Zahra Behroozi $^{1,2}$, Pezhman Atefimanesh ${ }^{3}$, Fariba Karimzadeh ${ }^{3 *}$}

${ }^{1}$ Shefa Neuroscience Research Center, Khatam Alanbia Hospital, Tehran, Iran

${ }^{2}$ Department of Physiology, Iran University of Medical Sciences, Tehran, Iran

${ }^{3}$ Cellular and Molecular Research Center, Iran University of Medical Sciences, Tehran, Iran

\section{A BSTRACT}

Introduction: Multiple Sclerosis (MS) is a chronic progressive course of illness that leads to the widespread pathological processes of neurodegeneration, including neuroaxonal damage, apoptosis and gliosis. Due to complex mechanisms involve in the pathophysiology of MS, finding valid biomarkers is very difficult. However, finding the effective biomarkers that are more sensitive and be able to determine the level of activity and course of the disease is necessary. Today, several biomarkers with high efficacy have been introduced to design the initial therapy, to evaluate the response to drug treatments, and to identify early diagnosis and different stages of the disease. This study was aimed to review different known biomarkers to diagnose the MS. Conclusion: The complicity of MS and the important role of biomarkers in the diagnosis of MS focus lead to several studies to evaluate the most effective biomarkers. The findings indicate that the cerebrospinal fluid (CSF) biomarkers are more valid compared to plasma biomarkers. CSF biomarkers are not influenced by the circadian rhythm and circulate in the subarachnoid space, which is in close proximity to sites of neuroinflammatory lesions in MS. Among structural biomarkers, magnetic resonance imaging is the most practicable methods for MS diagnosis.

\section{Key words:}

1. Multiple Sclerosis

2. Biomarkers

3. Spinal cord

*Corresponding Author: Fariba Karimzadeh

E-mail: kaimzade.f@iums.ac.ir 
نشانگر هاى زيستى ساختارى و متابوليتى در مالتيِل اسكلروز

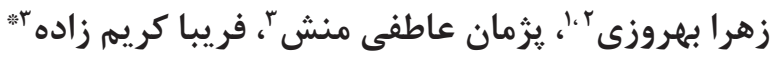 \\ 'مركز تحقيقات علوم اعصاب شفا، بيمارستان خاتمالانبيا، تهران، ايران \\ rَّروه فيز يولوزى، دانشخاه علوم يزشكى ايران، تهران، ايران

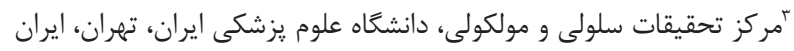

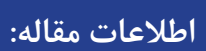

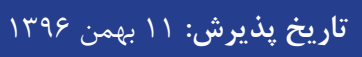

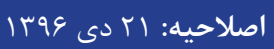

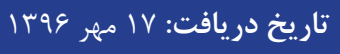

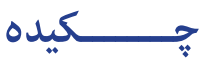

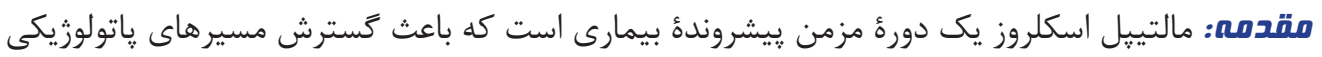

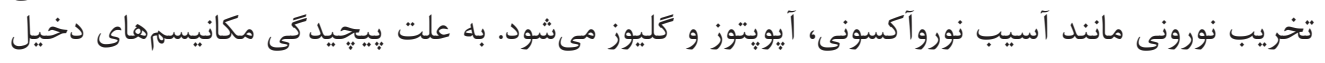

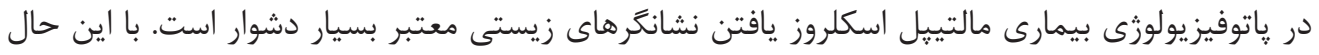

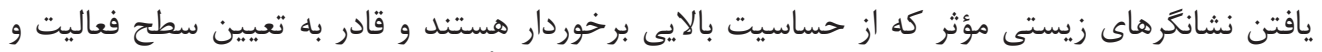

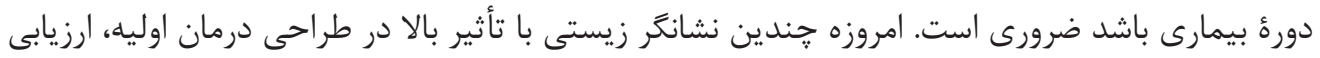

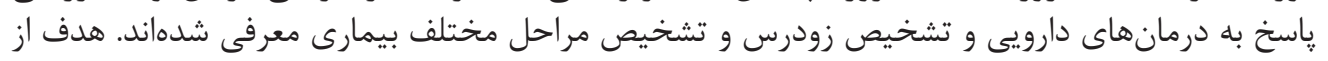

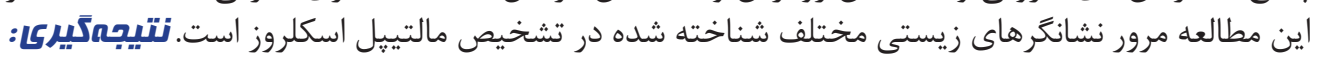

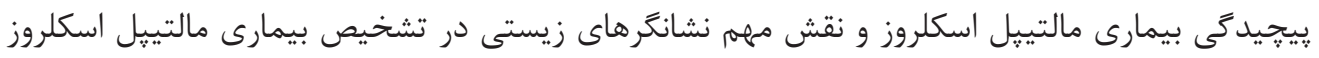

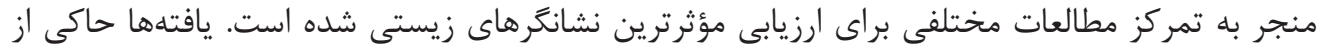

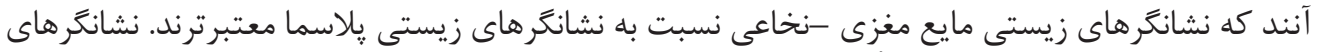

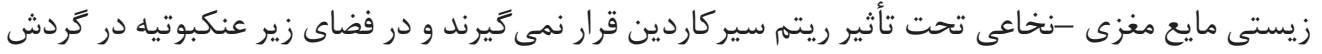

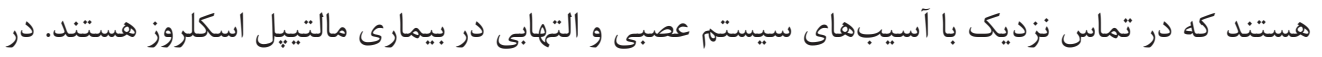

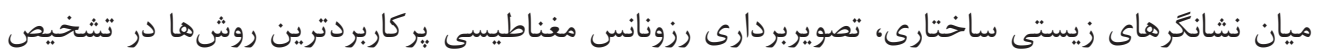
بيمارى مالتييل اسكلروز مىباشند.

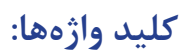

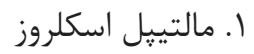

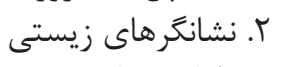

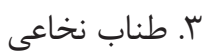

" نويسنده مسئول: فريبا كريم زاده آدرس الكترونيكى: kaimzade.f@iums.ac.ir 
زمــان، عـود يــا بهبـودى مشـخصى ديــده نمى شـود و بيمـار

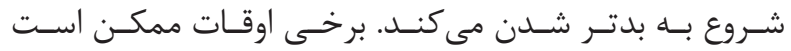

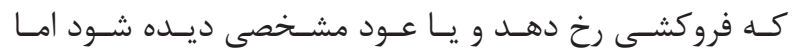

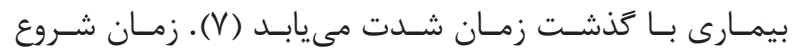

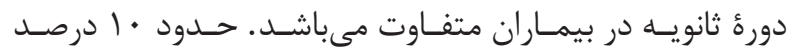

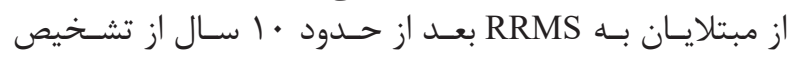

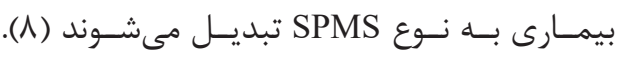

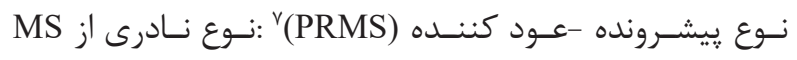

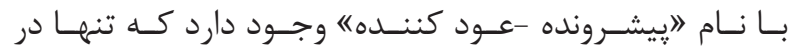

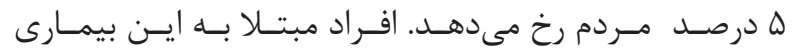

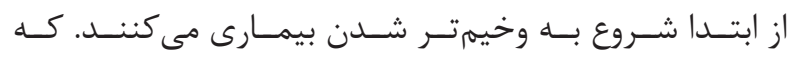

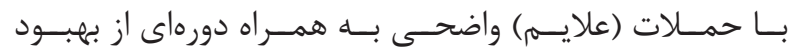

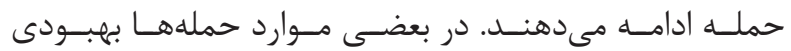

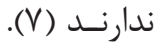

علايسم بالينى

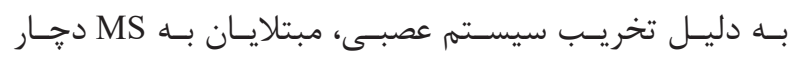

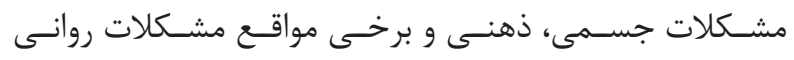

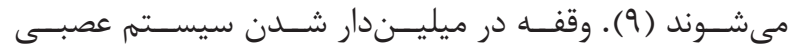

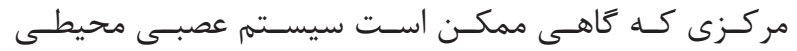

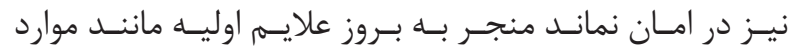

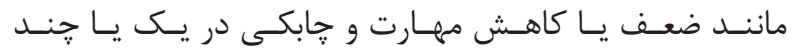

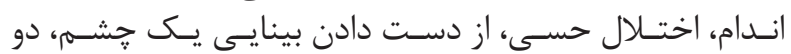

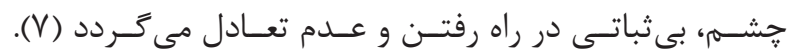

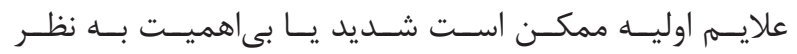

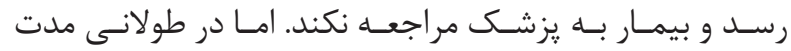

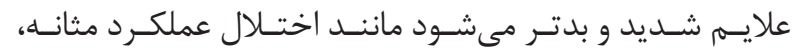

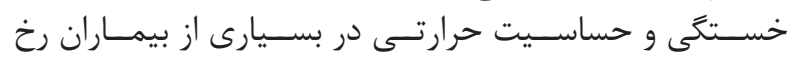

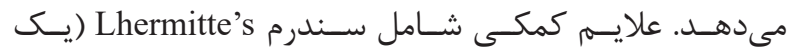

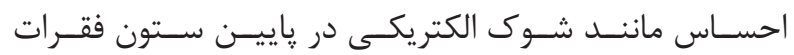

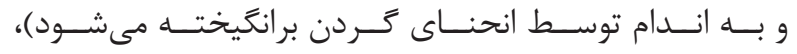

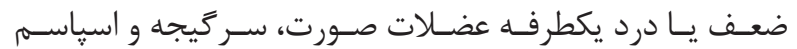

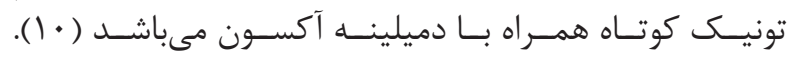

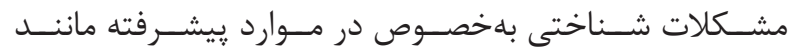

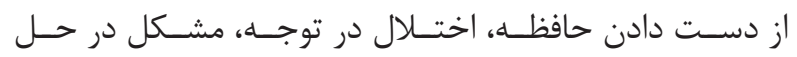

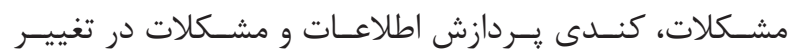

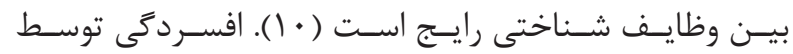

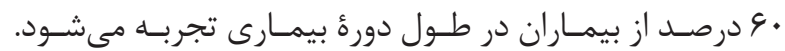

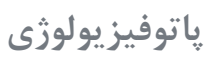

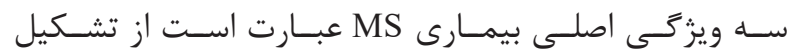

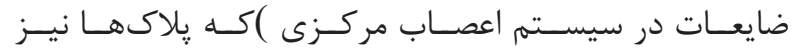

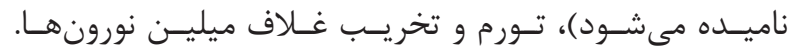

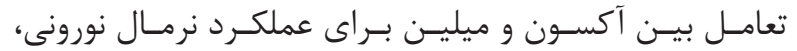

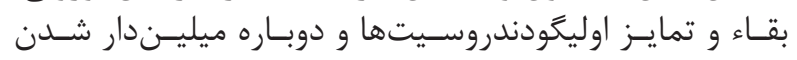

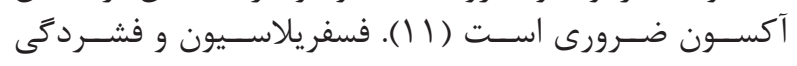

${ }^{1}$ Multiple sclerosis

${ }^{2}$ Morphology

${ }^{3}$ Relapsing -remitting MS

${ }^{4}$ Remission

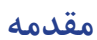

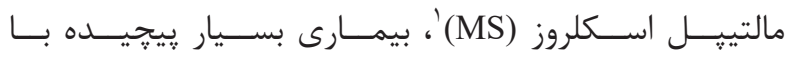

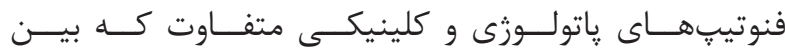

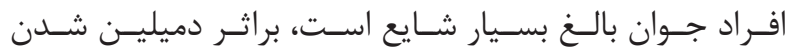

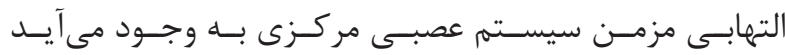

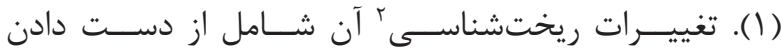

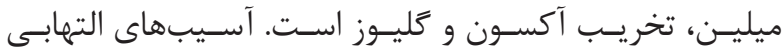

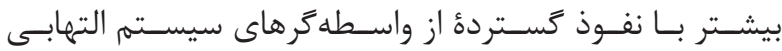

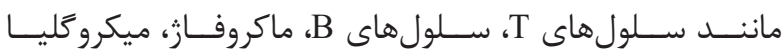

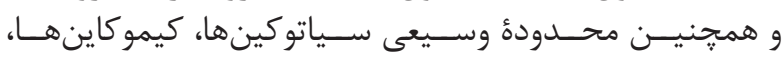

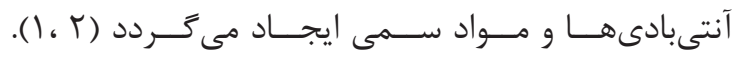

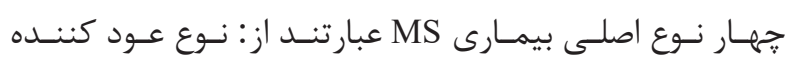

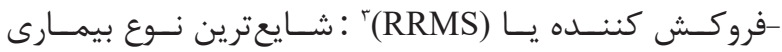
MS

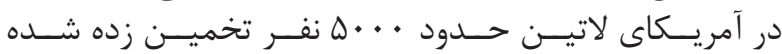

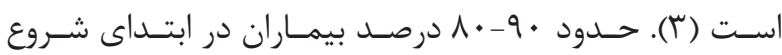

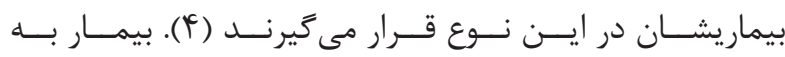

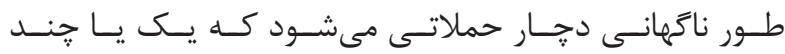

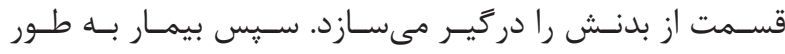

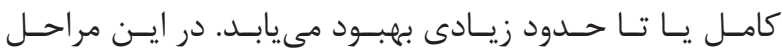

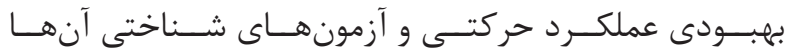

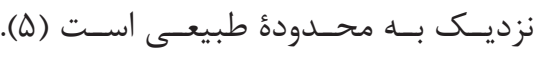

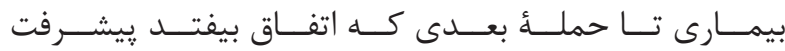

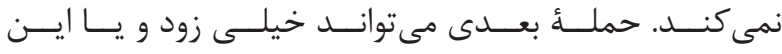

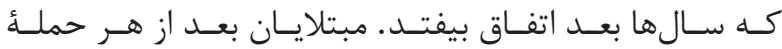

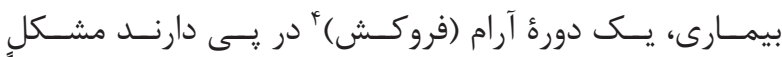

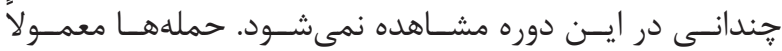

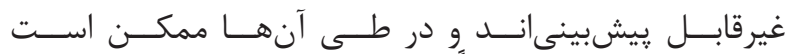

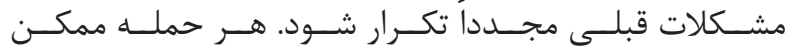

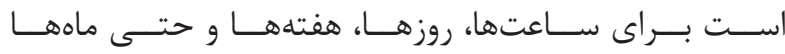

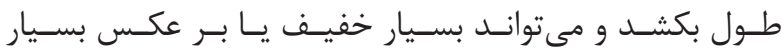

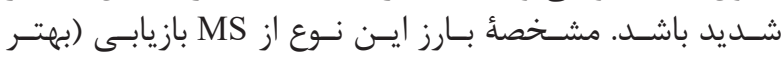

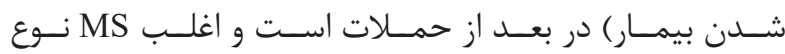

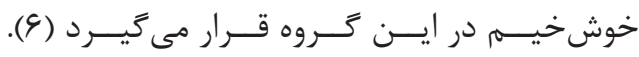

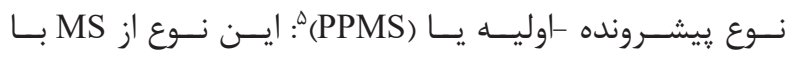

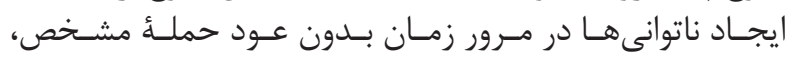

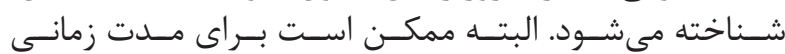

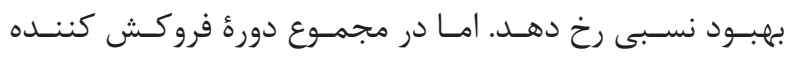

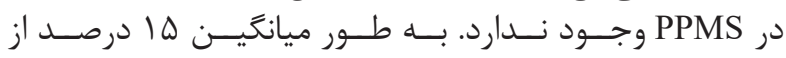

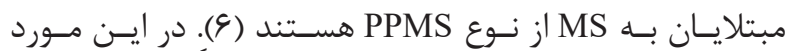

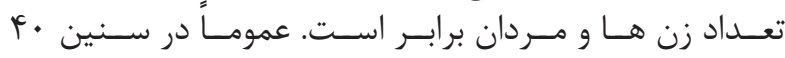

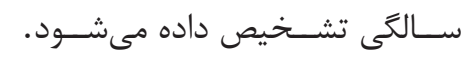

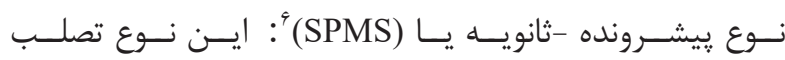

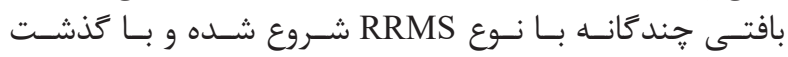

${ }^{5}$ Primary progressive MS

${ }^{6}$ Secondary progressive MS

${ }^{7}$ Progressive relapsing MS 


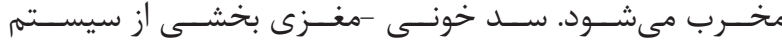

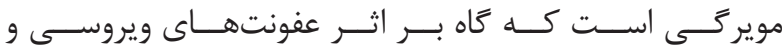

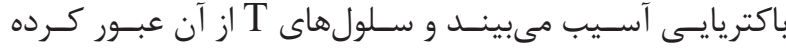

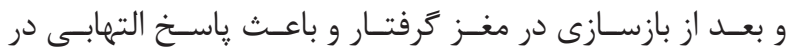

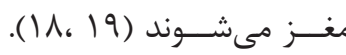

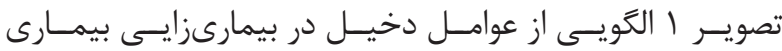
MS

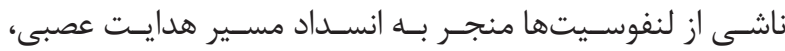

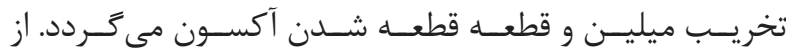

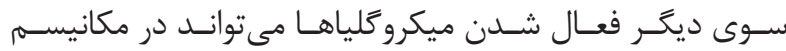

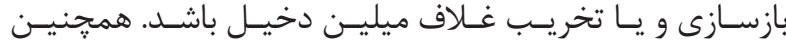

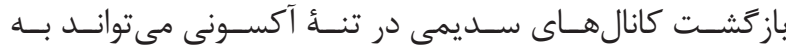

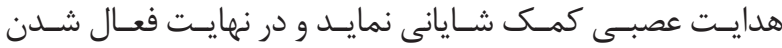

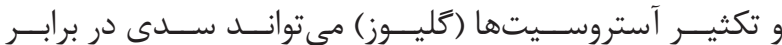

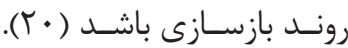

MS تشخيص بيمارى

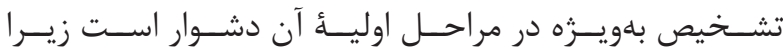

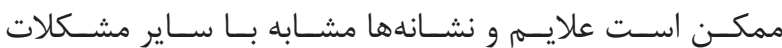

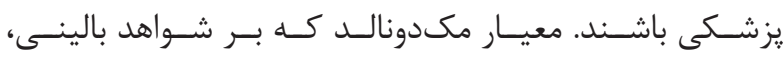

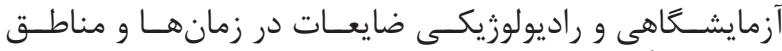

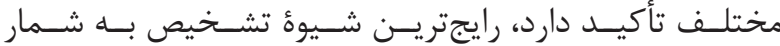

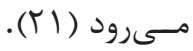

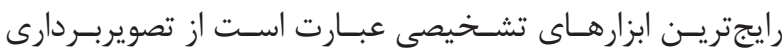

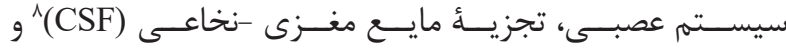

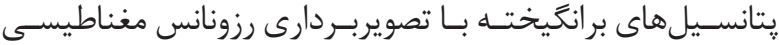

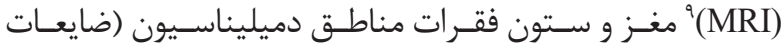

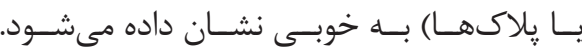

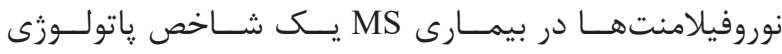

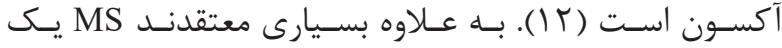

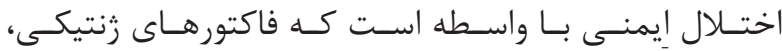

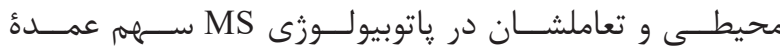

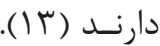

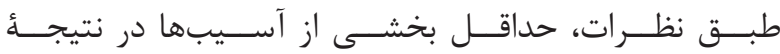

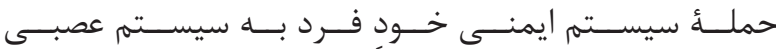

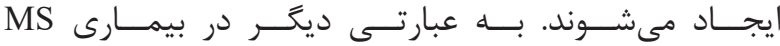

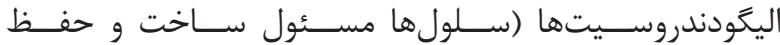

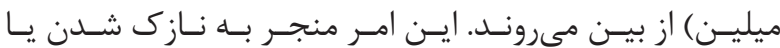

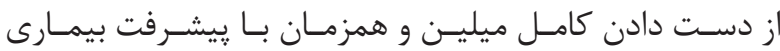

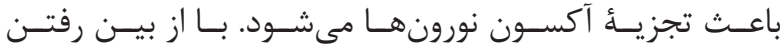

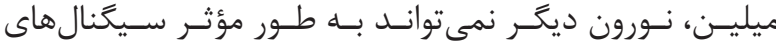

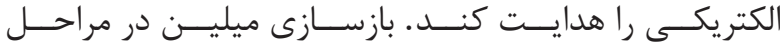

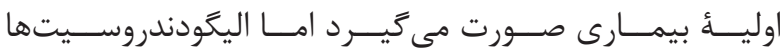

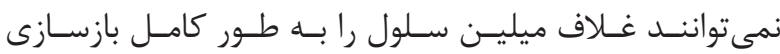

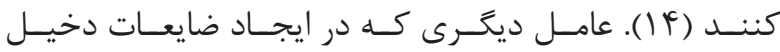

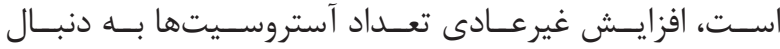

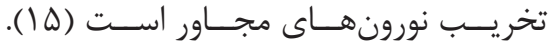

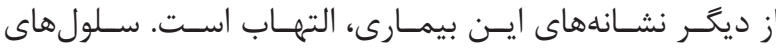

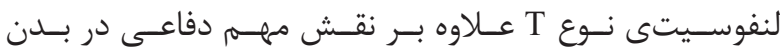

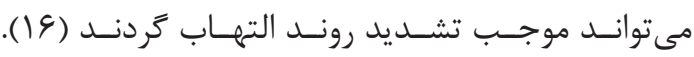

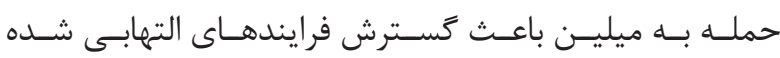

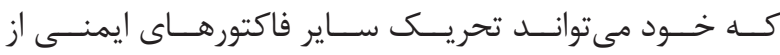

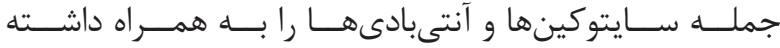

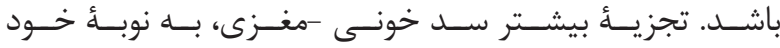

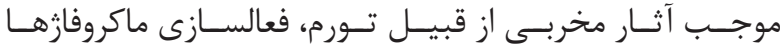

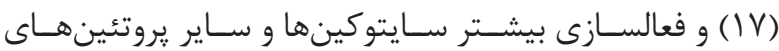

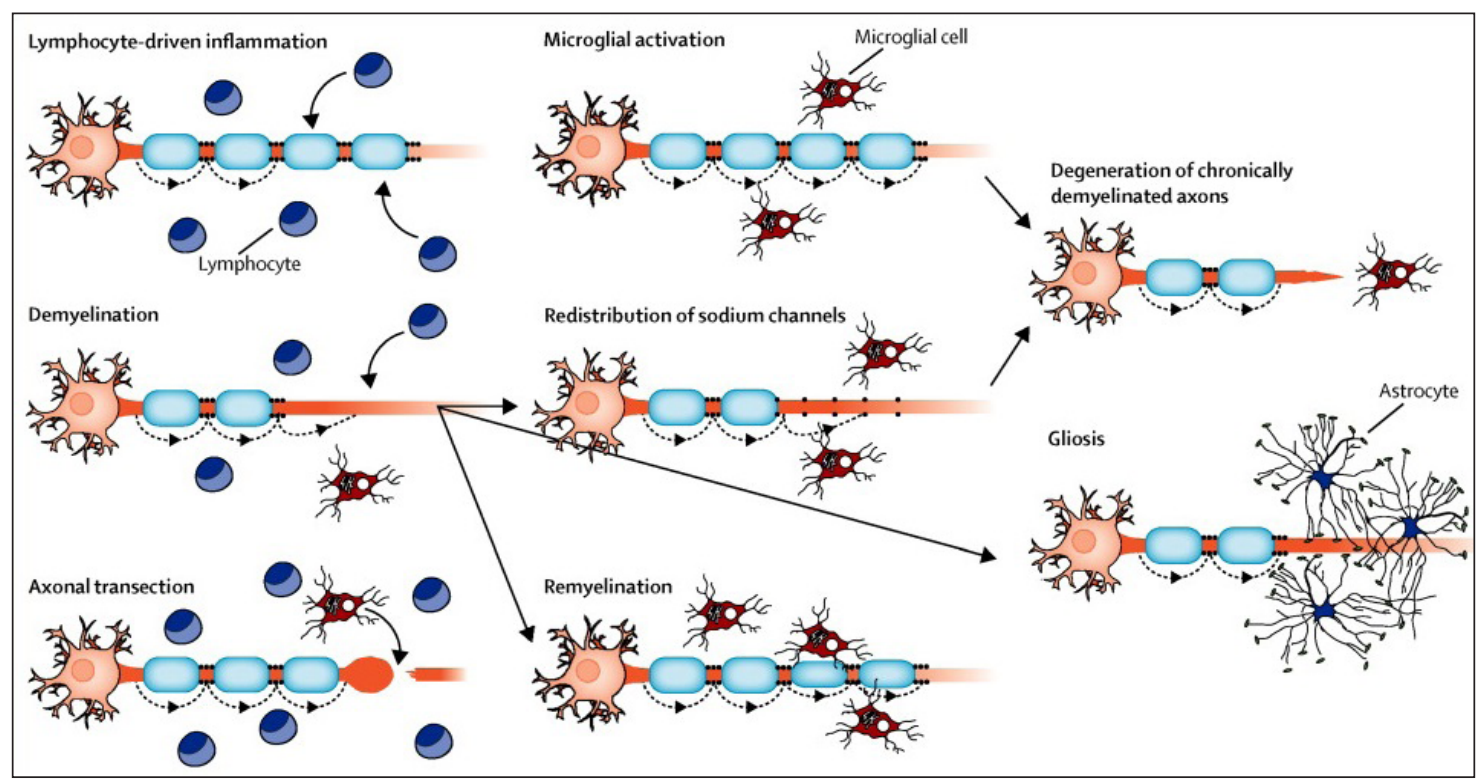

تصوير ا- الكَيى از سير پاتولوريك در بيمارى MS (†).

${ }^{8}$ Cerebrospinal fluid

${ }^{9}$ Magnetic resonance imaging 


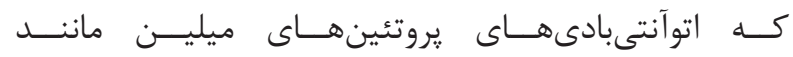

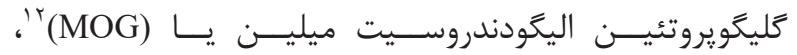

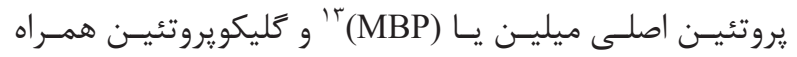

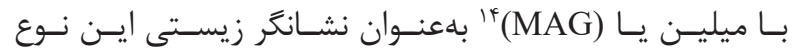

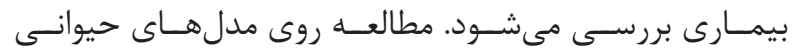

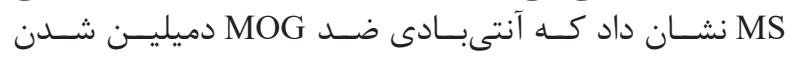

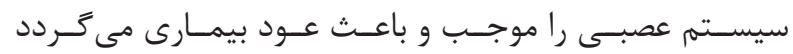
(YN) MS

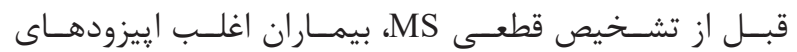

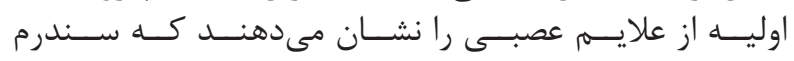

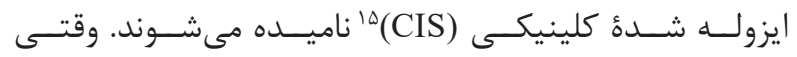

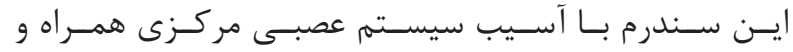

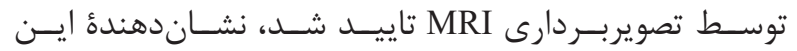

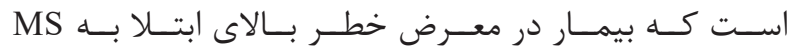

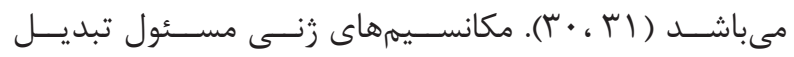

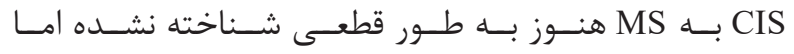

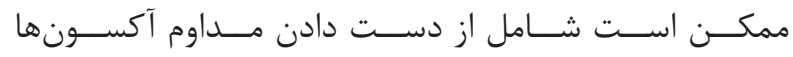

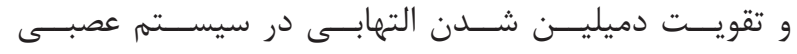

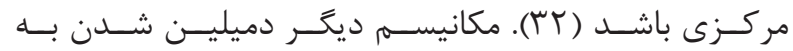

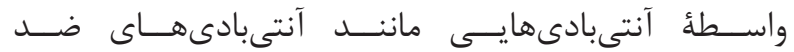

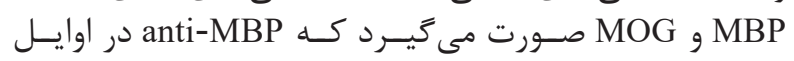

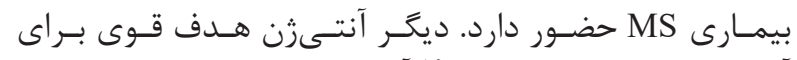

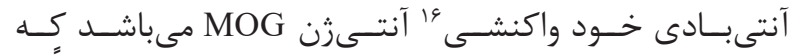

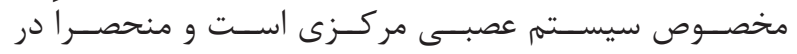

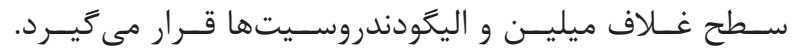

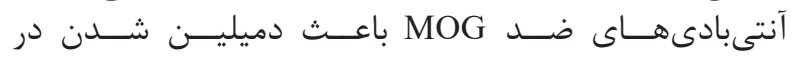

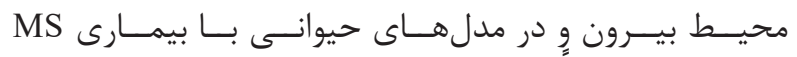

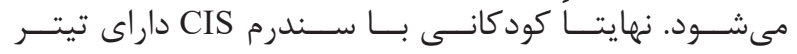

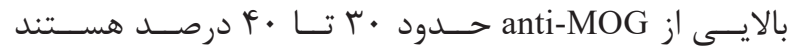

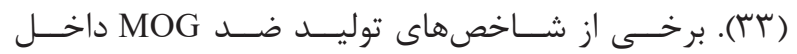

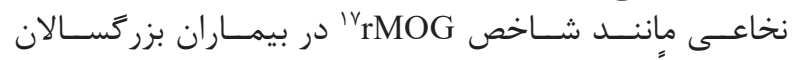

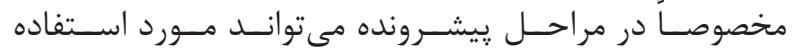

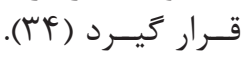

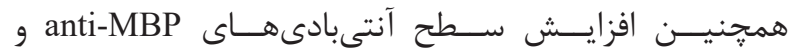
anti- MOG

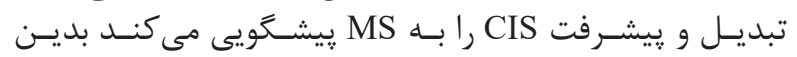

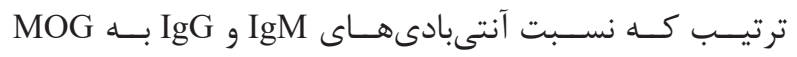

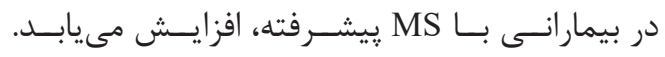

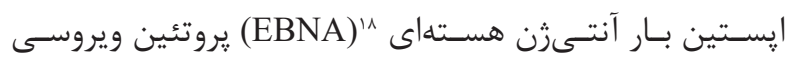

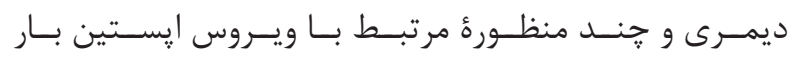
(EBV)

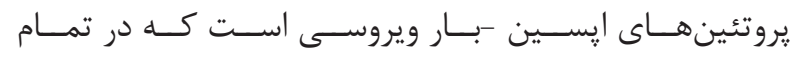

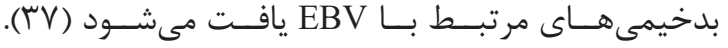

\footnotetext{
${ }^{10}$ Dawson's fingers

${ }^{11}$ Biomarker

${ }^{12}$ Myelin oligodendrocyte glycoprotein

${ }^{13}$ Myelin basic protein

${ }^{14}$ Myelin associated glycoprotein
}

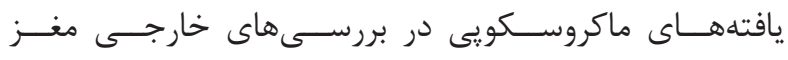

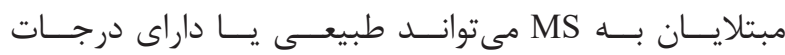

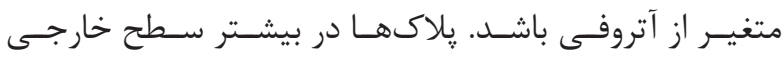

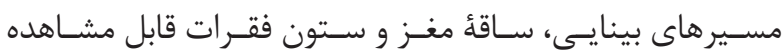

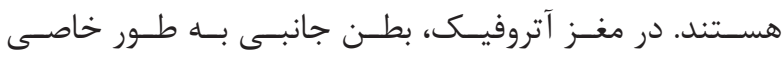

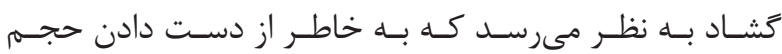

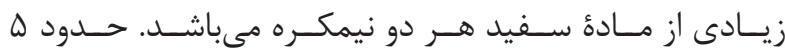

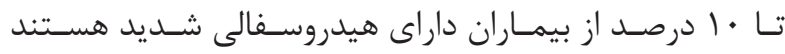

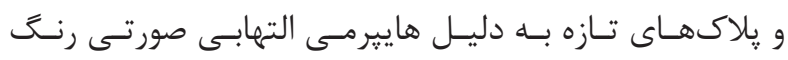

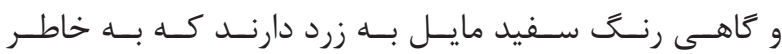

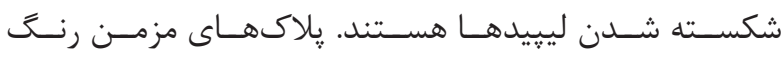

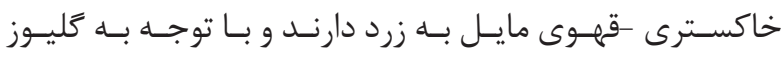

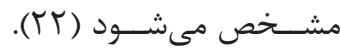

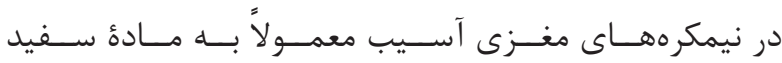

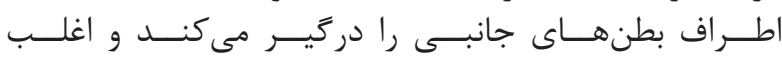

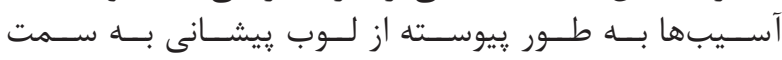

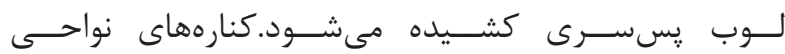

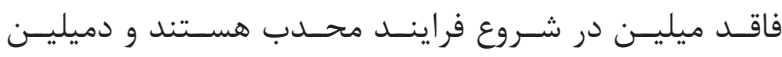

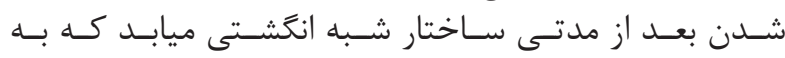

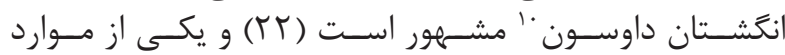

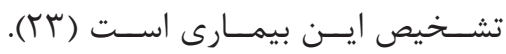

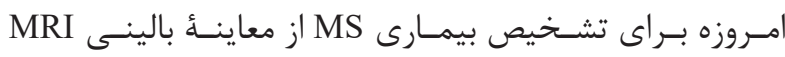

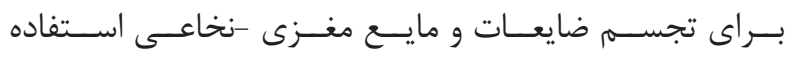

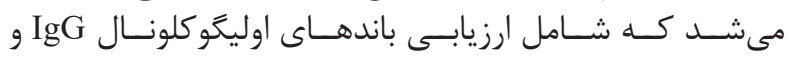

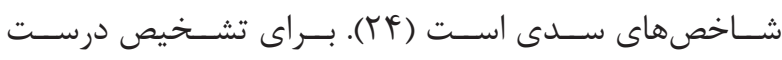

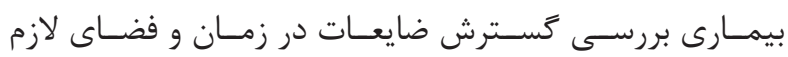

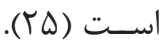

\section{نشانگر زيستى ״يست؟}

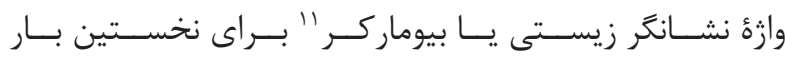

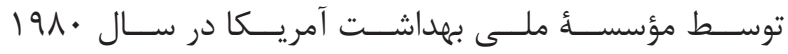

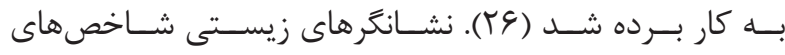

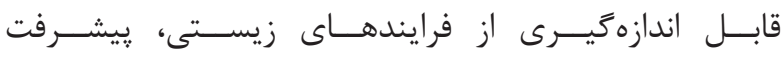

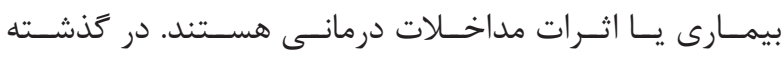

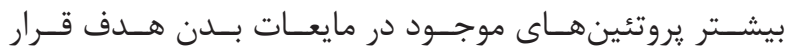

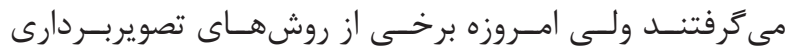

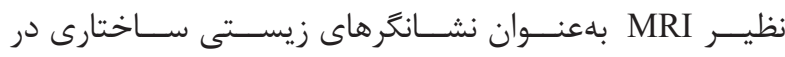

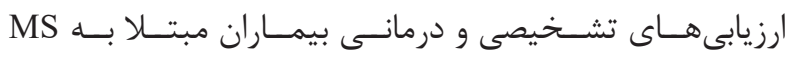

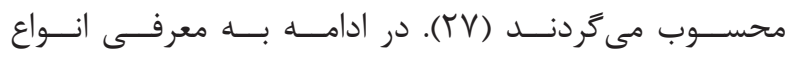

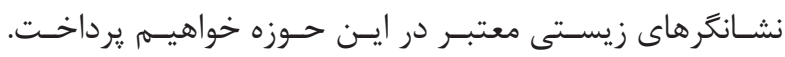
نشانًَر هاى زيستى مايع مغزى -نخاعى

آنتىبادى هاى ضد ميلين

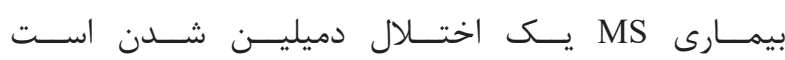

${ }^{15}$ Clinically isolated syndrome

${ }^{16}$ Autoreactive antibodies

${ }^{17}$ Recombinant MOG

${ }^{18}$ Epstein-Barr nuclear antigen

${ }^{19}$ Epstein-Barr virus 


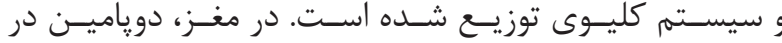

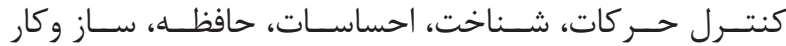

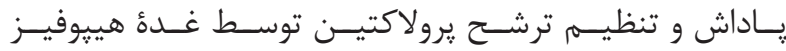

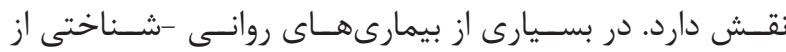

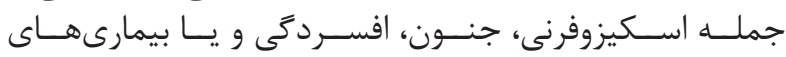

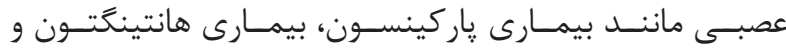

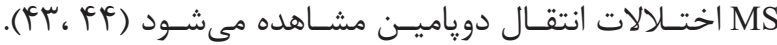
افزايسش سـطح ايسن :ِروتئيسن در سـلولهاى كليـال مبتلايـان

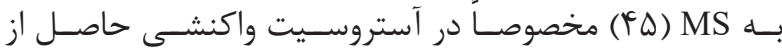

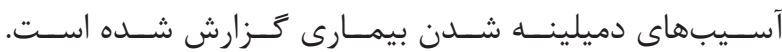

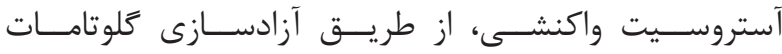

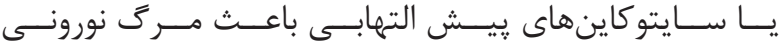

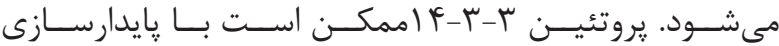

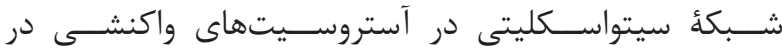

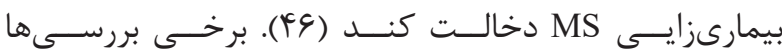

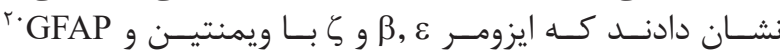

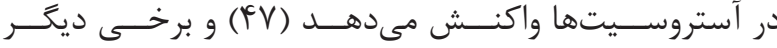

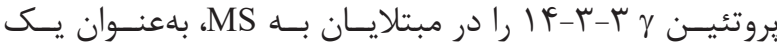

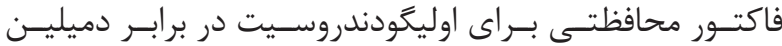

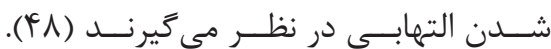

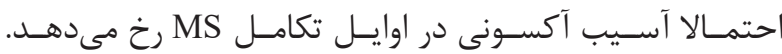

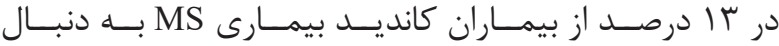

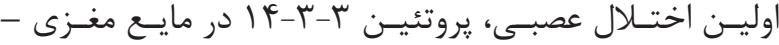

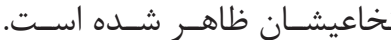

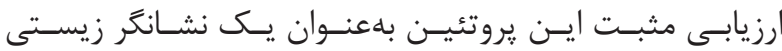

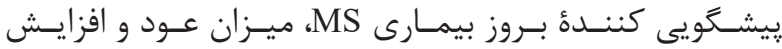

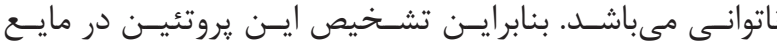

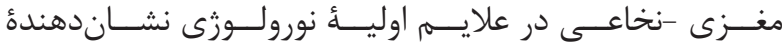

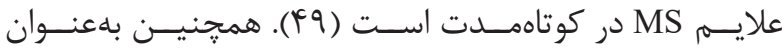

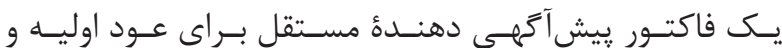

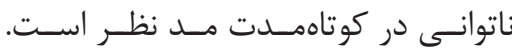

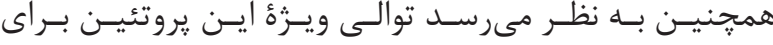

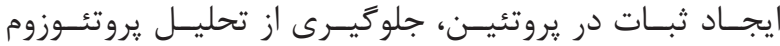

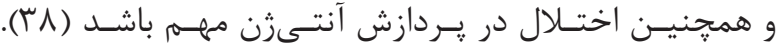

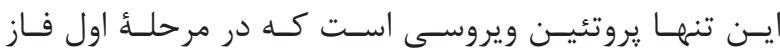

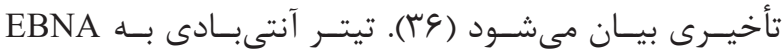

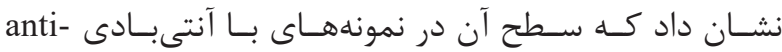

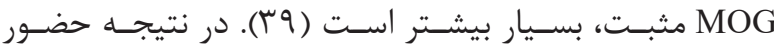

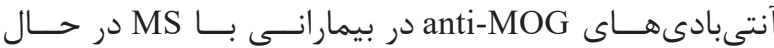

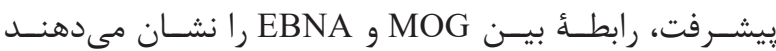

\section{بروتئين r-r}

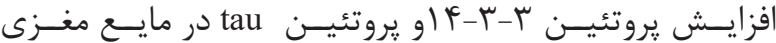

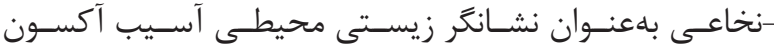

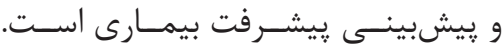

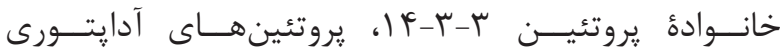

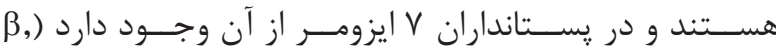

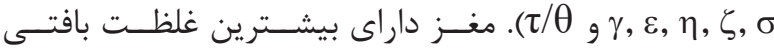

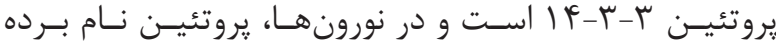

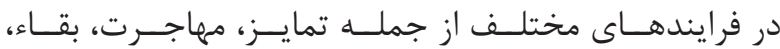

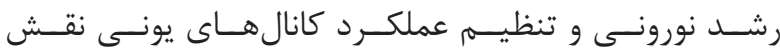

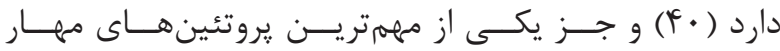

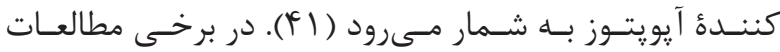

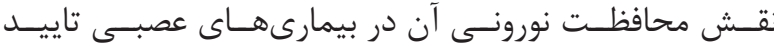

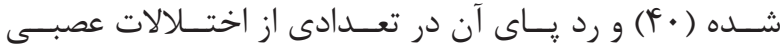

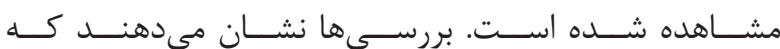

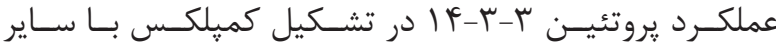

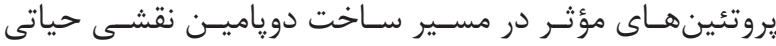

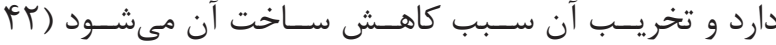

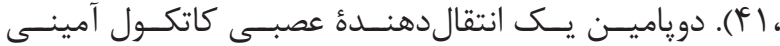

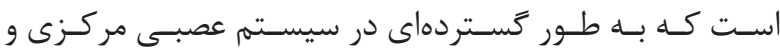

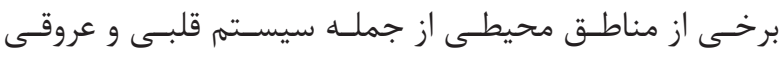

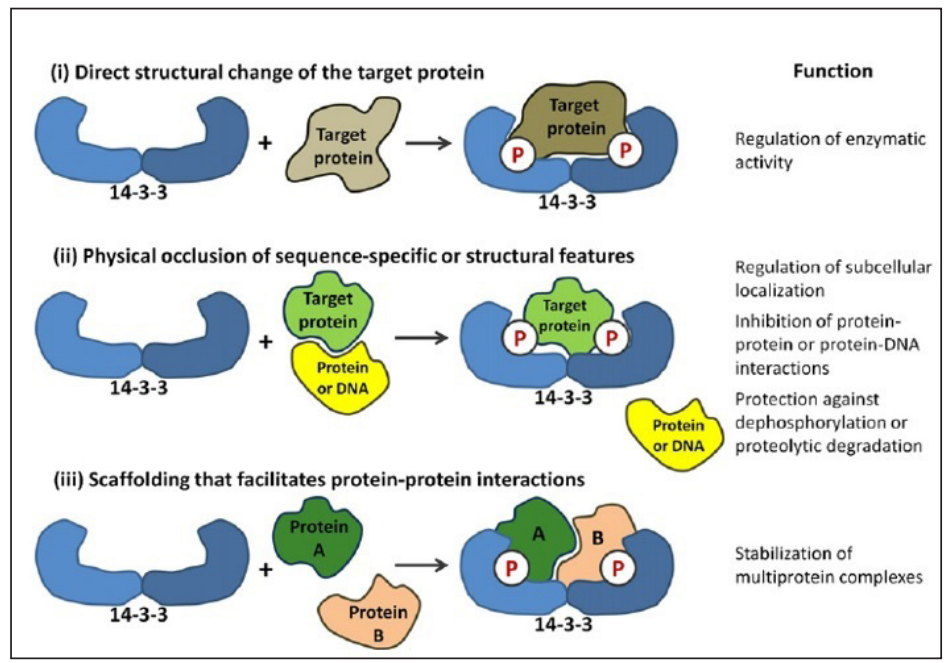

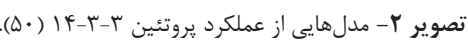

${ }^{20}$ Glial fibrillary acidic protein 


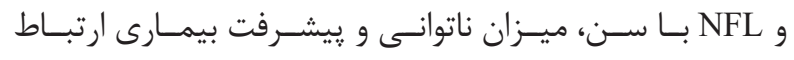

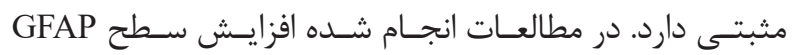

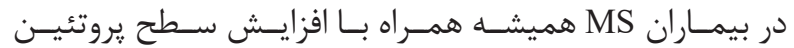

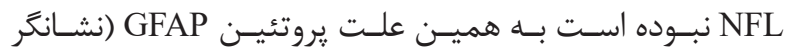

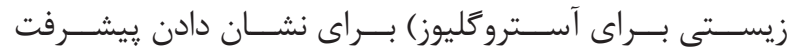

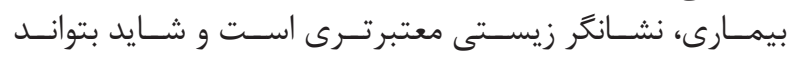

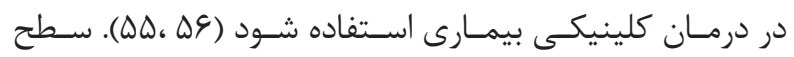

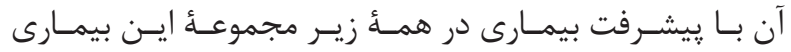

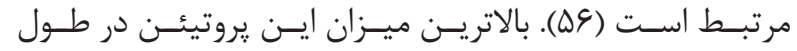

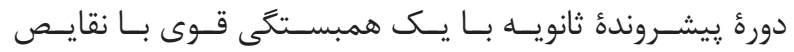

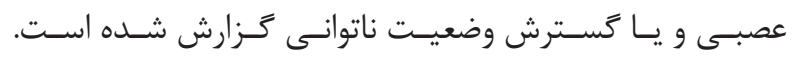

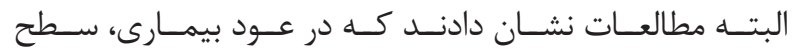

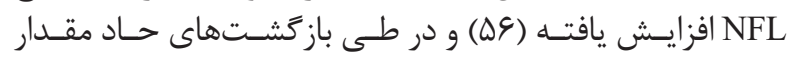

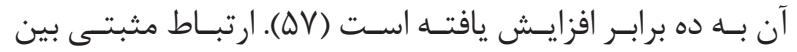

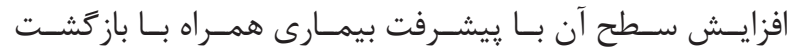

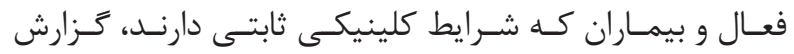

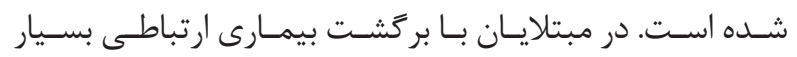

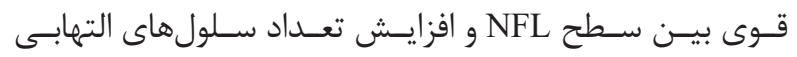
وجـود دارد (DS).

\section{Cئئين سيستاتين C}

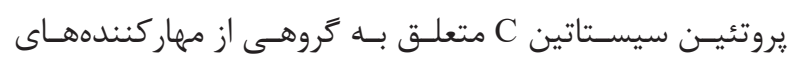

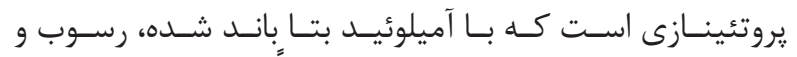

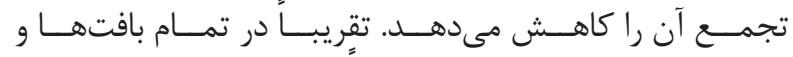

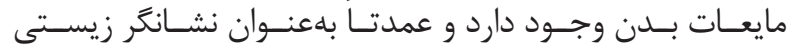

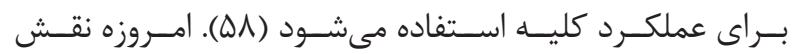

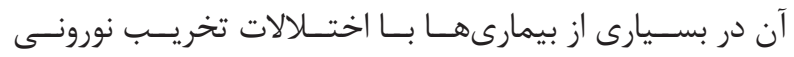

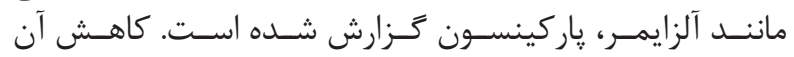

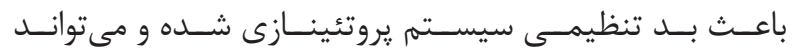

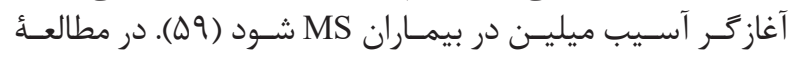

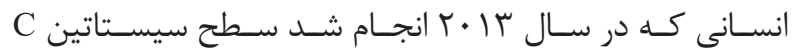

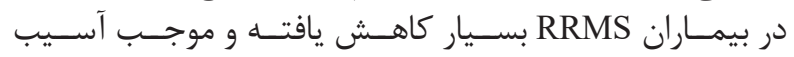

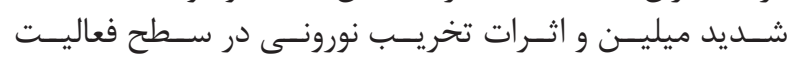

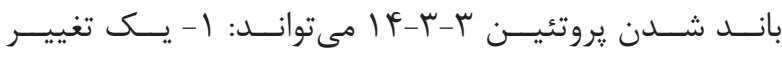

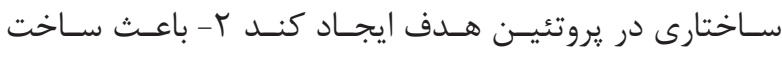

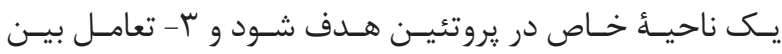

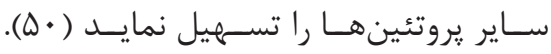

Tau بروتئين

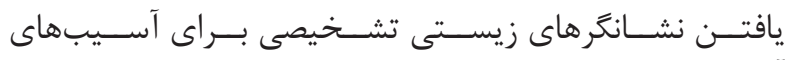

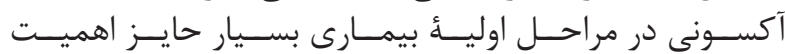

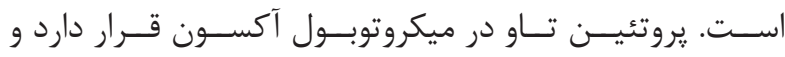

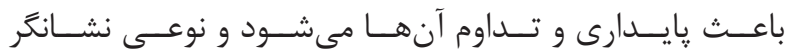

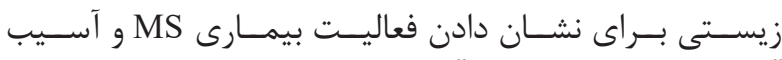

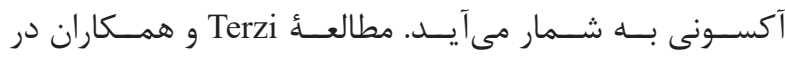

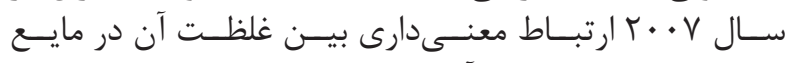

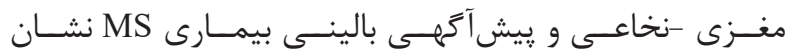

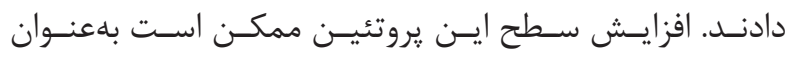

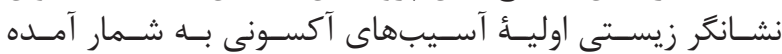

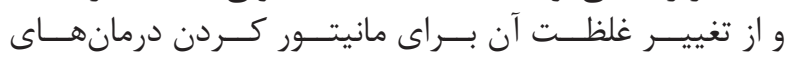

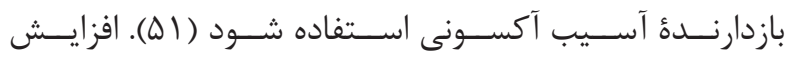

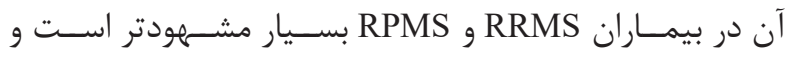

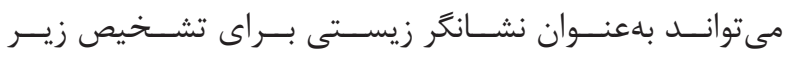

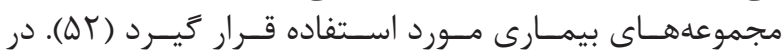

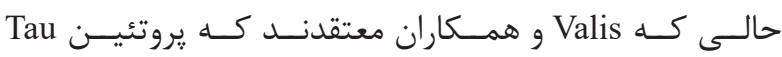

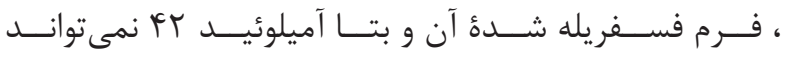

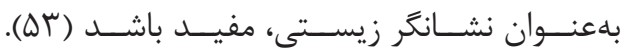

\section{يروتئينهاى GFAP و NFL}

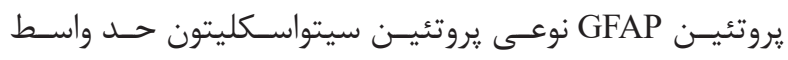

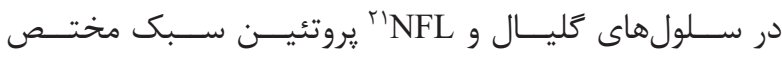

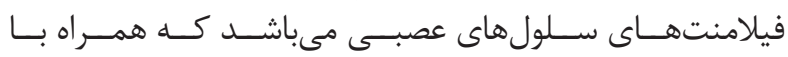

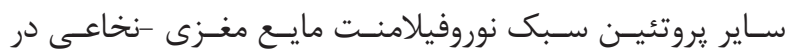

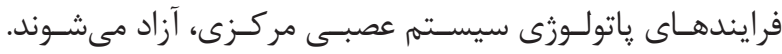

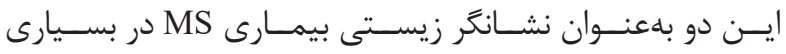

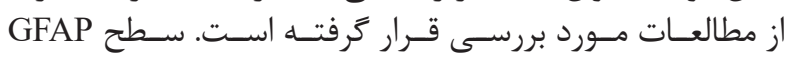

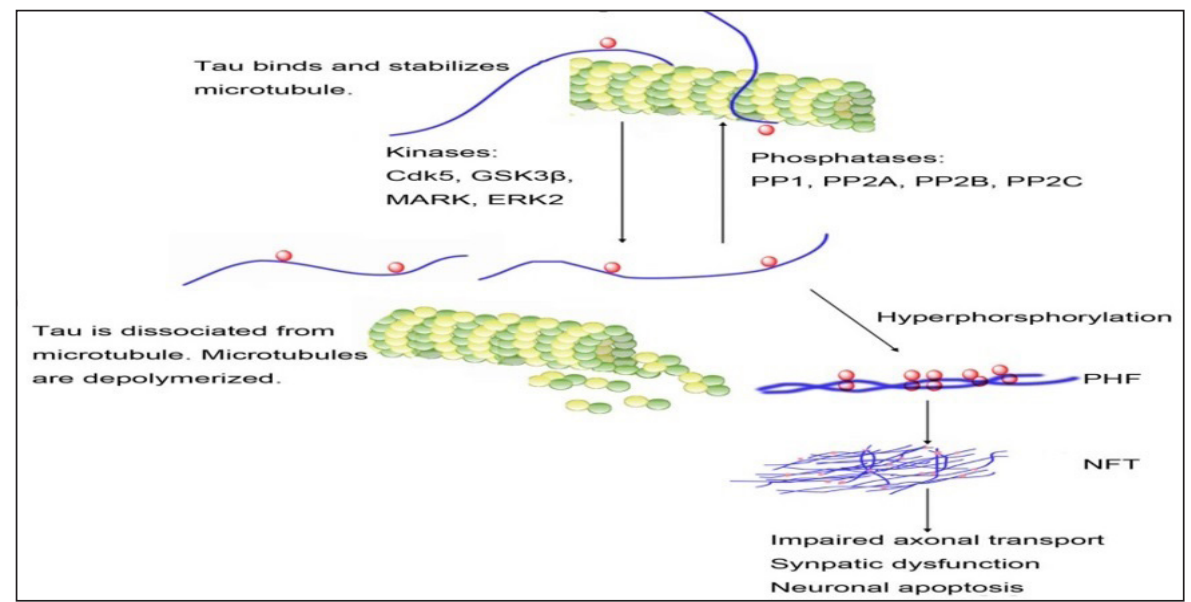

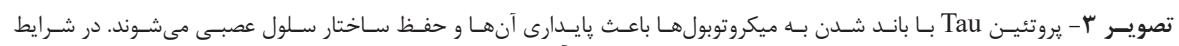

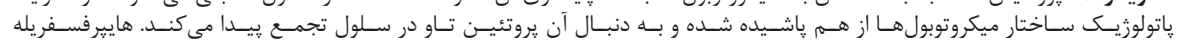

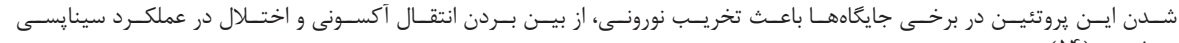

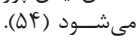

${ }^{21}$ Neurofilament light protein 


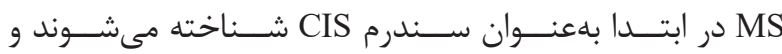

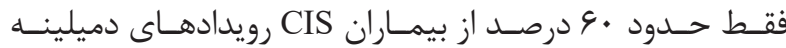

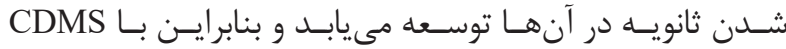

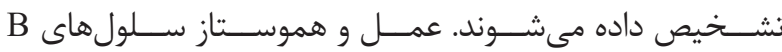

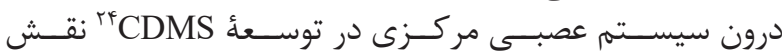

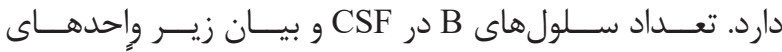

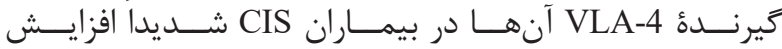

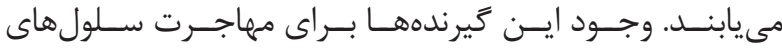

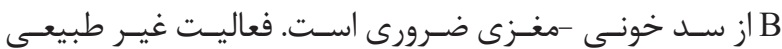

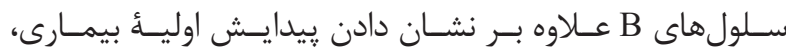

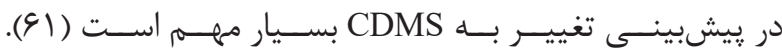

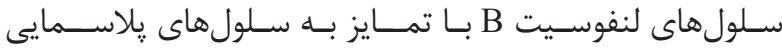

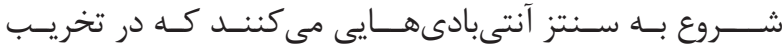

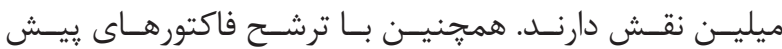

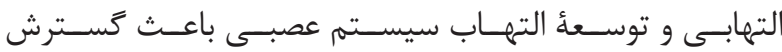

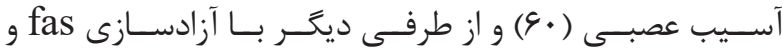

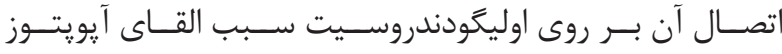

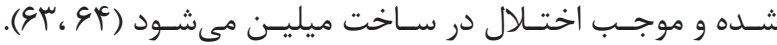

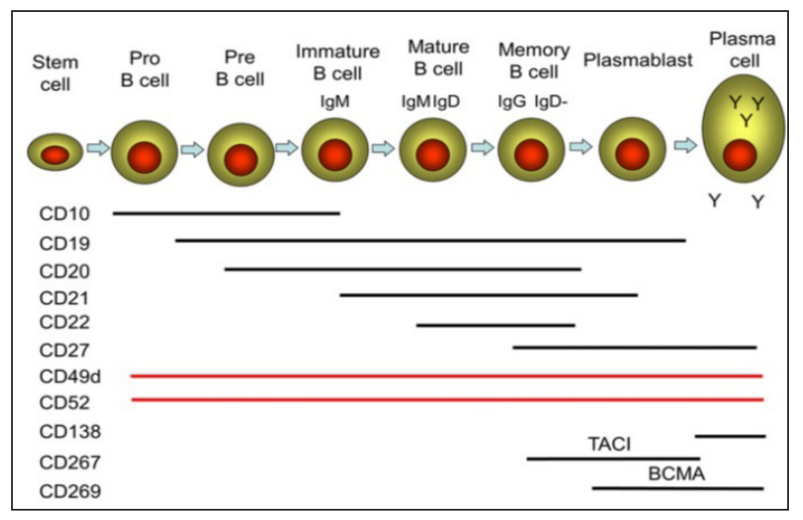

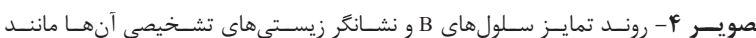
(\$Q) B cell maturation antigen :BCMA

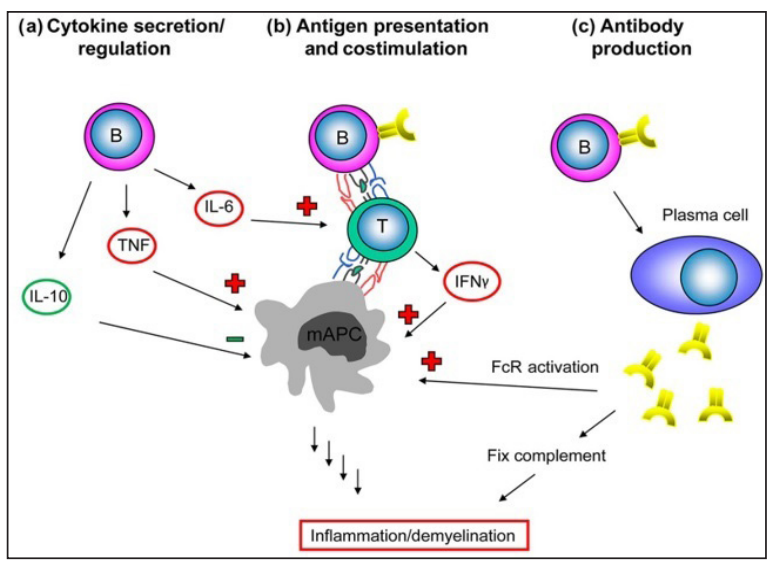

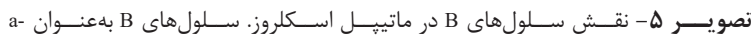

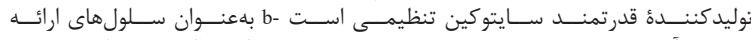

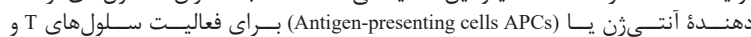

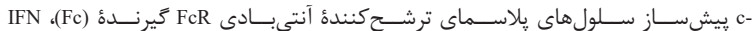

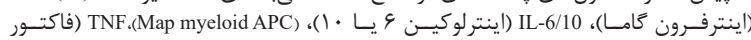

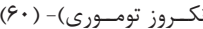

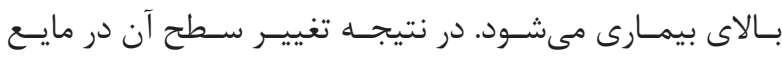

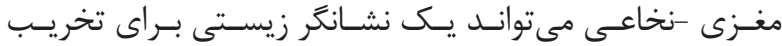

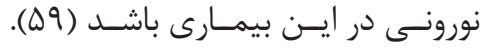

لنفوسيتهاى

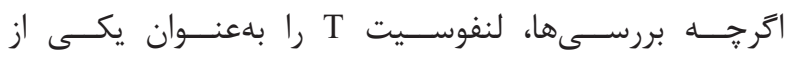

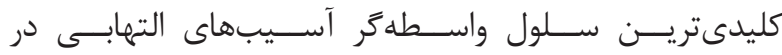

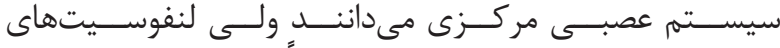

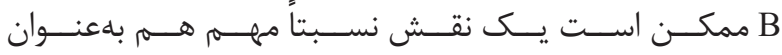

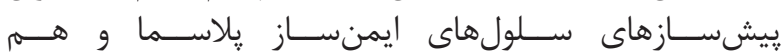

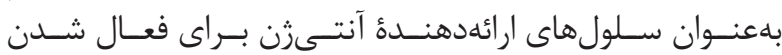

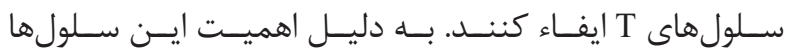

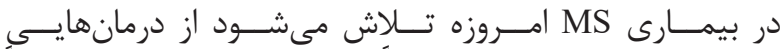

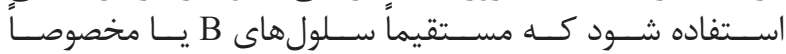

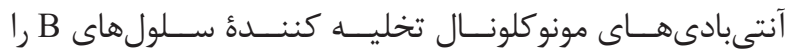

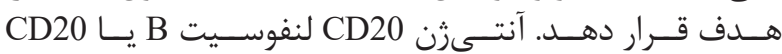

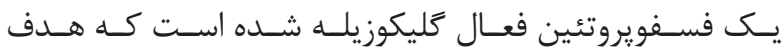

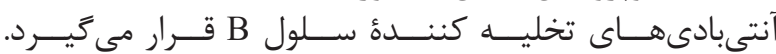

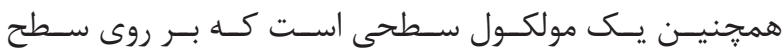

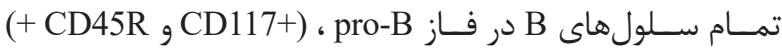

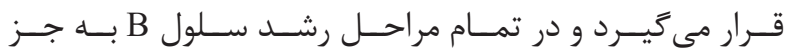

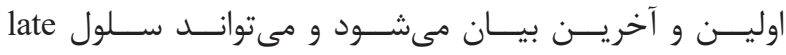

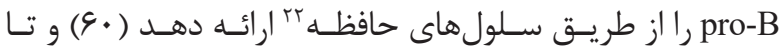

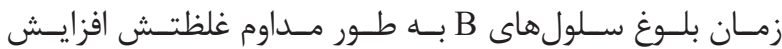

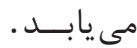

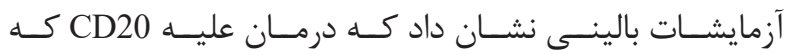

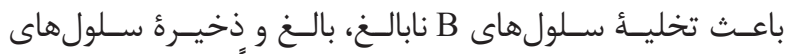

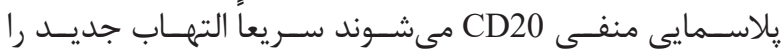

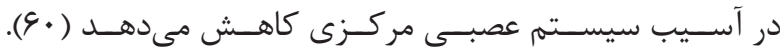

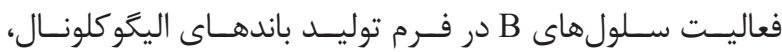

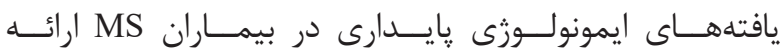

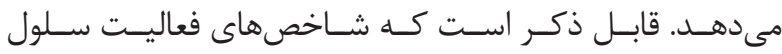

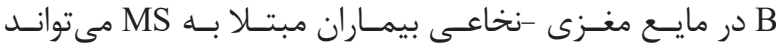

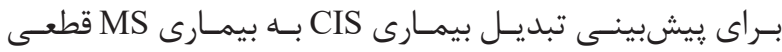

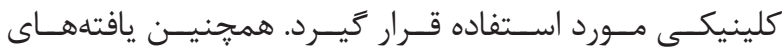

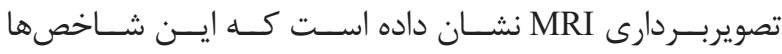

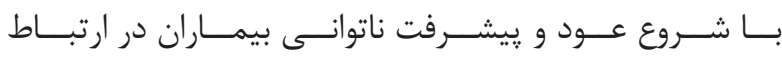

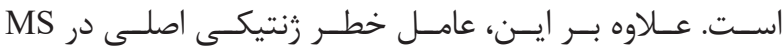

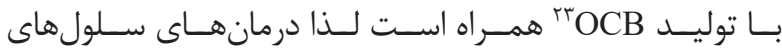

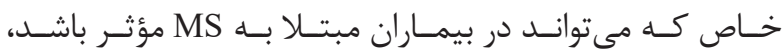

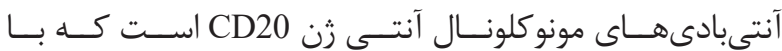

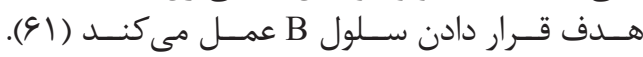

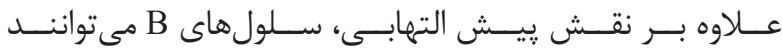

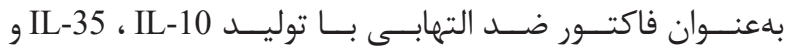

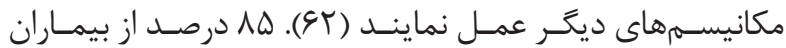

${ }^{22}$ Memory cells

${ }^{23}$ Oligoclonal bands

${ }^{24}$ Clinically definite MS 
جدول ا- ساير نشانكر هاى زيستى CSF به اختصار.

\begin{tabular}{|c|c|}
\hline \multicolumn{2}{|r|}{ نشانكرهاى زيستى ايمنوكّلوبولينهاى CSF } \\
\hline OCB IgM & 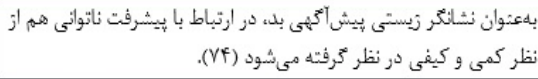 \\
\hline OCB IgG & 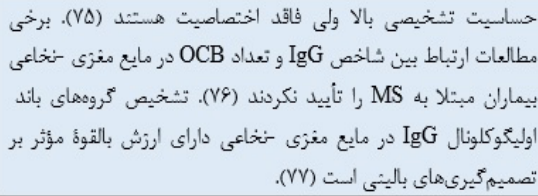 \\
\hline $\begin{array}{l}\text { Kappa Free Light } \\
\text { Chains (KFLC) }\end{array}$ & 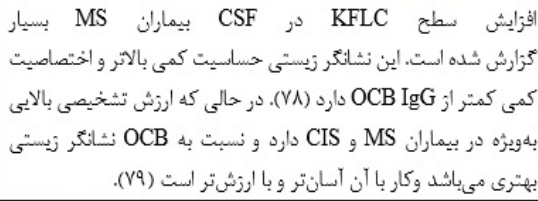 \\
\hline $\begin{array}{l}\text { آنثيوتانسيثورن } \\
\text { (ANGT) }\end{array}$ & 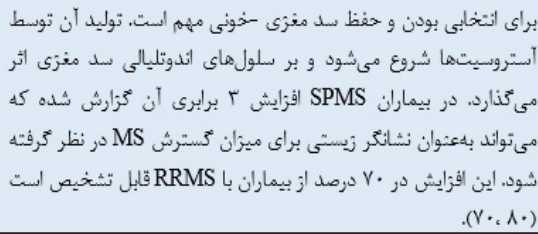 \\
\hline $\begin{array}{l}\text { Vascular } \\
\text { endothelial growth } \\
\text { factor-A (VEGF-A) }\end{array}$ & 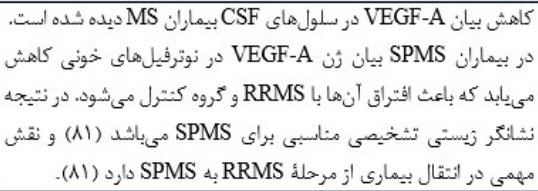 \\
\hline $\begin{array}{l}\text { Vitamin D-binding } \\
\text { protein (DBP) }\end{array}$ & 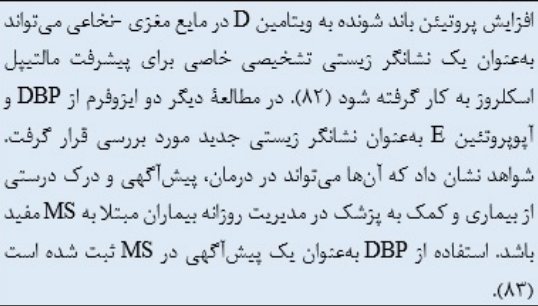 \\
\hline
\end{tabular}

$$
\text { نشانَّر هاى زيستى هيلاسما و سرم }
$$

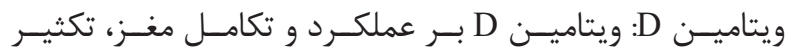

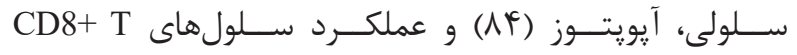

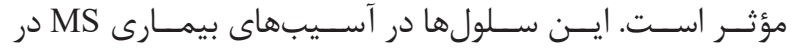

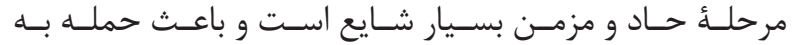

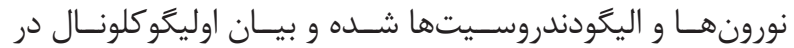

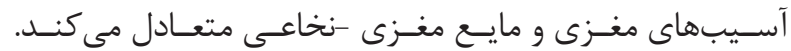

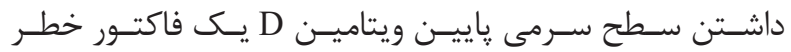

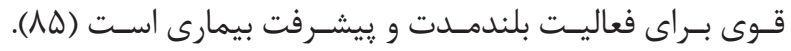

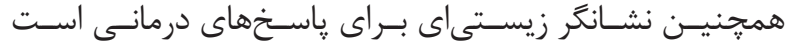

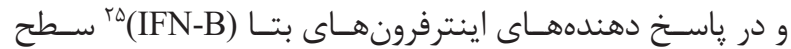

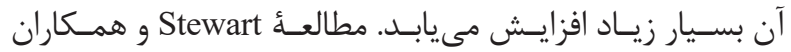

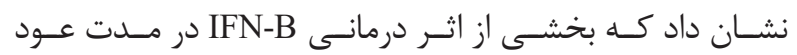

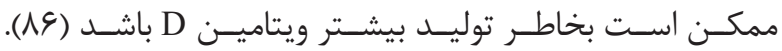

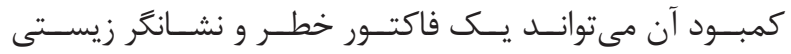

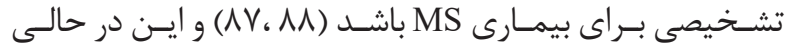

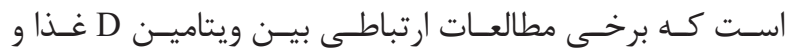

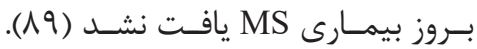

فيبولين

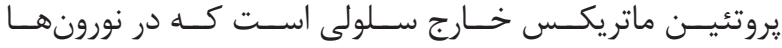

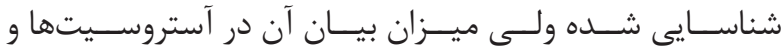

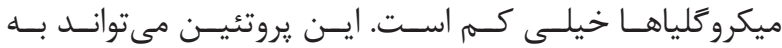

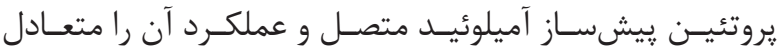

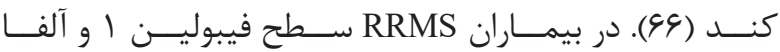

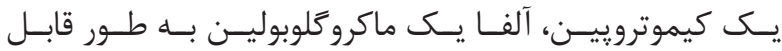

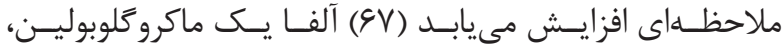

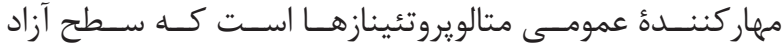

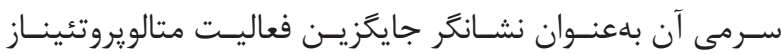

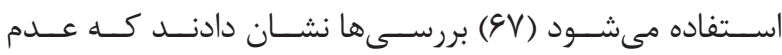

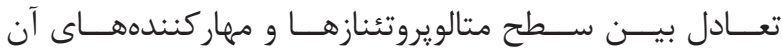

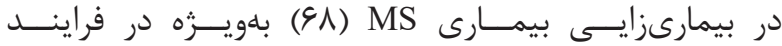

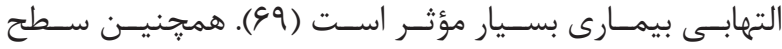

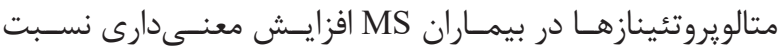

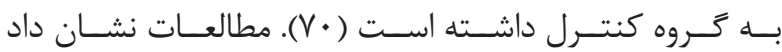

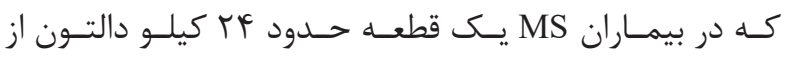

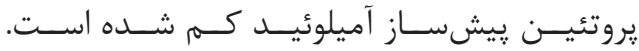

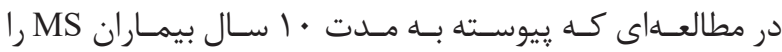

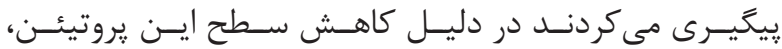

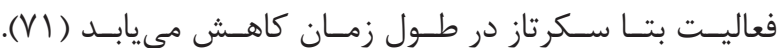

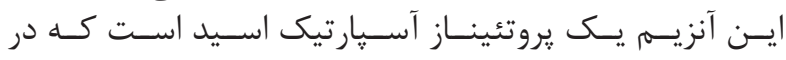

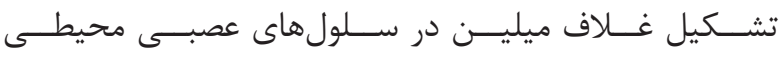
بسـيار مهـم اسـت (VT) (VT)

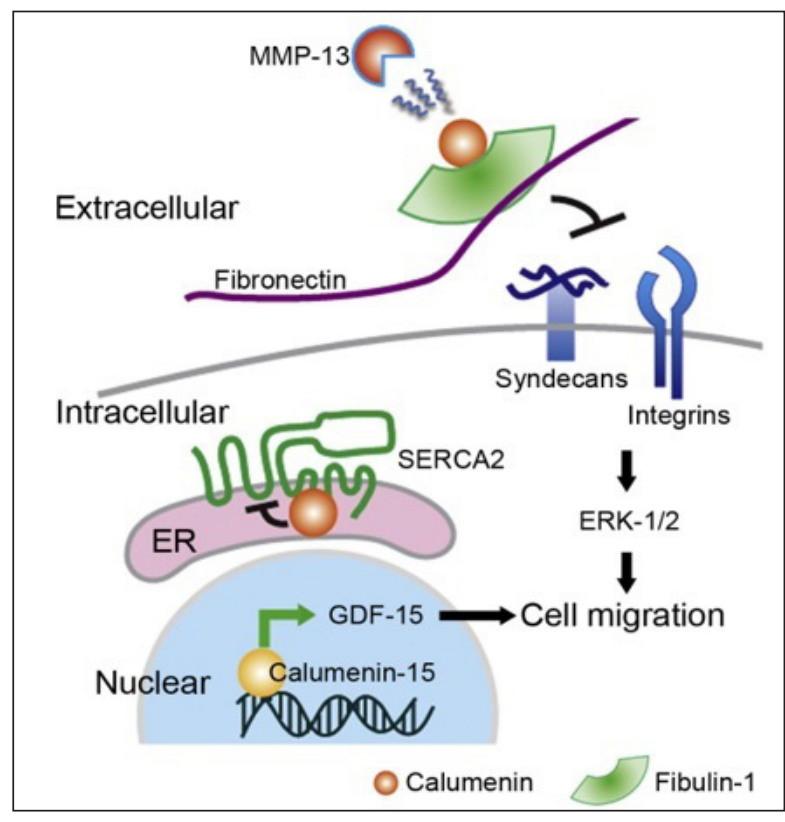

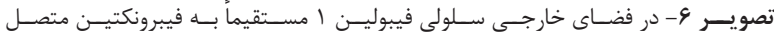

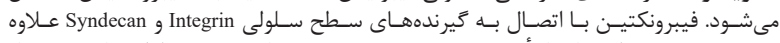

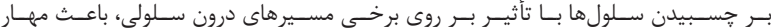

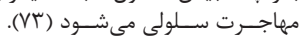

${ }^{25}$ Interferon beta 


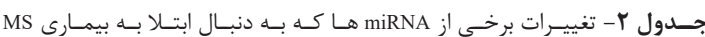

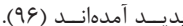

\begin{tabular}{|c|c|c|c|}
\hline miRNA & عيزان بيان & عملكرد & نشائكر زيستى \\
\hline $\mathrm{miR}-7-1-3 p$ & افزايش & & خون \\
\hline $\mathrm{miR}-15 \mathrm{~b}$ & كاهش & & سرم \\
\hline miR-20a-5p & كاهش & & خون \\
\hline miR-21 & اقزايش & افزايش Th17 و Th1 & خون \\
\hline miR-23a & كاهش & & خون \\
\hline miR-23b & افزايش & اقزايش Th17 & خون \\
\hline $\mathrm{miR}-27 \mathrm{~b}$ & افزايش & اقزايش Th1 & خون \\
\hline miR-92a-1 & اقزايش & & بلالسما \\
\hline miR-124 & افزايش & & \\
\hline miR-125a-5p & كاهش & & \\
\hline $\operatorname{miR}-128$ & افزايش & اقزايش Thl & \\
\hline miR-145 & افزايش & & صيلاسسما \\
\hline miR-146a & افزايش & الفزايش Th17 & \\
\hline miR-155 & الفزايش & اقزايش Th17 و Th17 & مِلاسما و مغز \\
\hline $\operatorname{miR}-223$ & كاهش & & سرم \\
\hline $\mathrm{miR}-326$ & اقزايش & الفزايش Th17 & \\
\hline $\operatorname{miR}-340$ & افزايش & اقزايش Th1 & \\
\hline let-7e & افزايش & افزايش Th1 و Th17 & \\
\hline
\end{tabular}

نتيجه كيرى

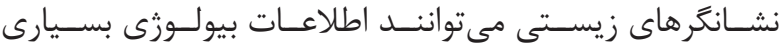

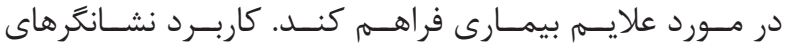

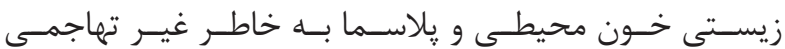

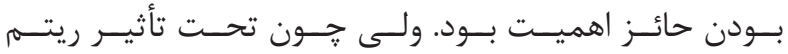

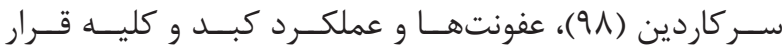

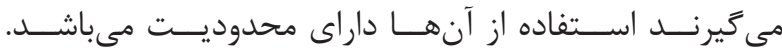

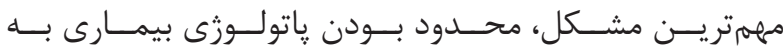

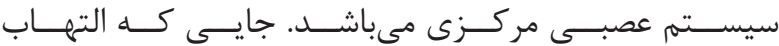

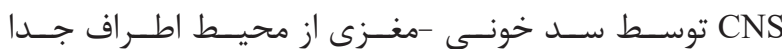

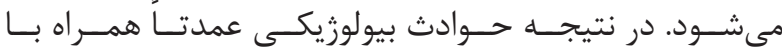

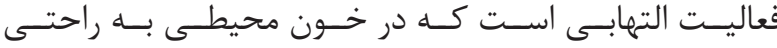

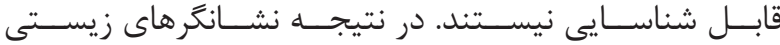

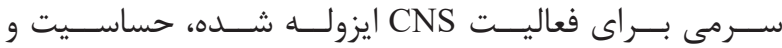

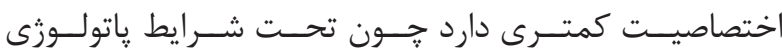

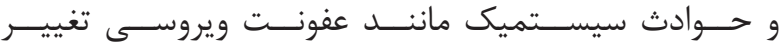

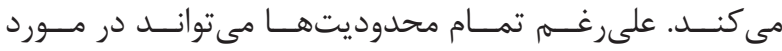

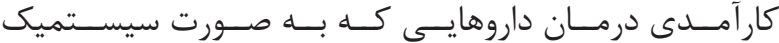

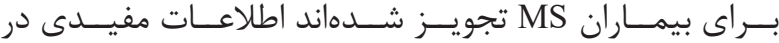

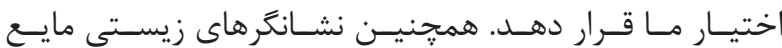

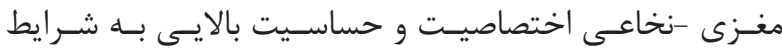

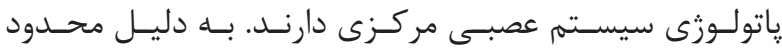

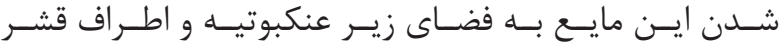

${ }^{26} 25$-hydroxyvitamin D

${ }^{27}$ miRNAs

${ }^{28} 3^{\prime}$ untranslated region

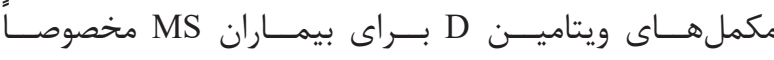

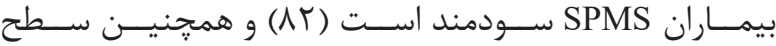

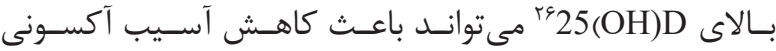
در بيمـاران MS تــردد (•) (9).

ميكرو RNA Rا

و و همــكاران (بـــهـ نقــل از Junker

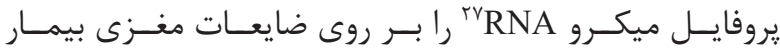

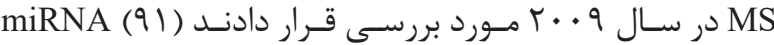

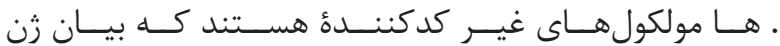

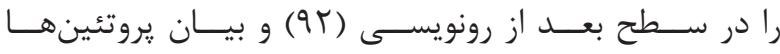

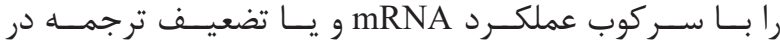

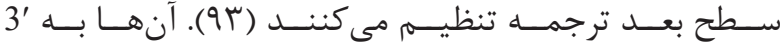

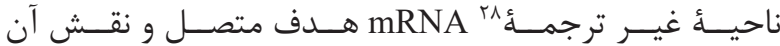

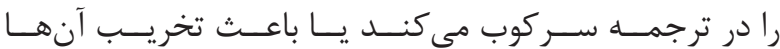

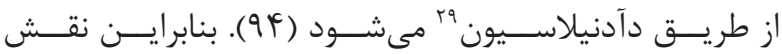

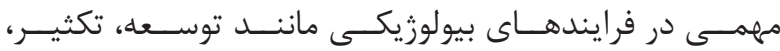

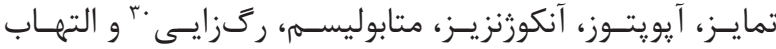

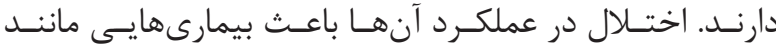

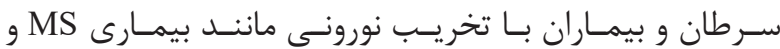

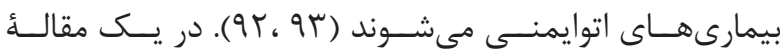

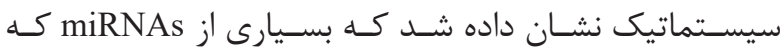

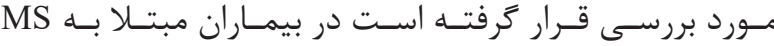

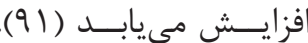

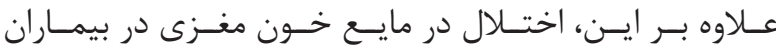

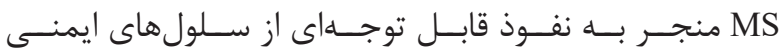

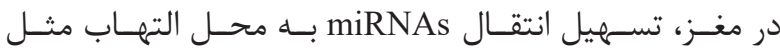

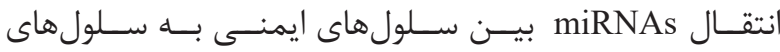
كليايــى مىشــود (94)

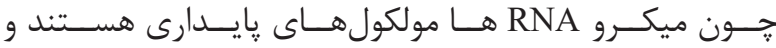

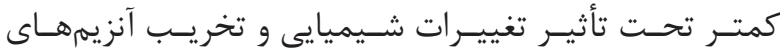

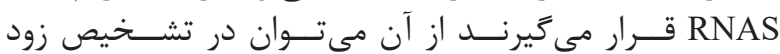

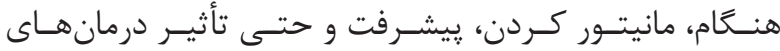

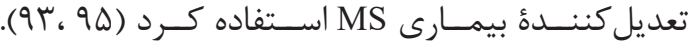

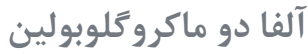

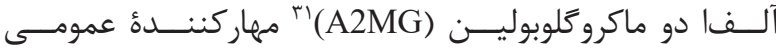

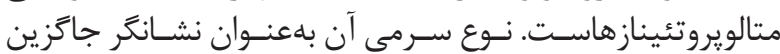

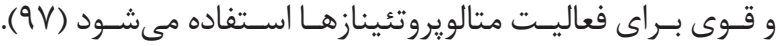

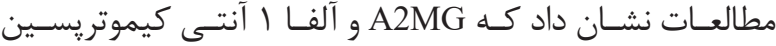

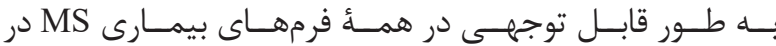

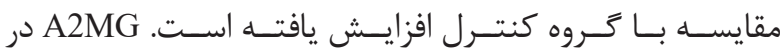

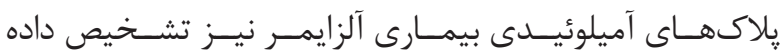

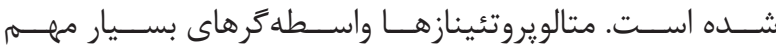

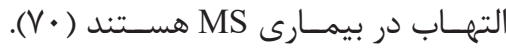

${ }^{29}$ Deadenylation

${ }^{30}$ Angiogenesis

${ }^{31}$ Alpha-2-Macroglobulin 


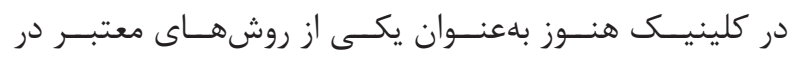

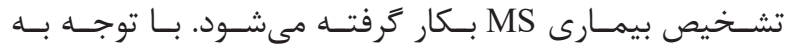

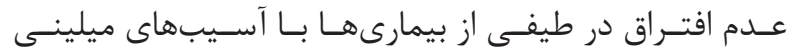

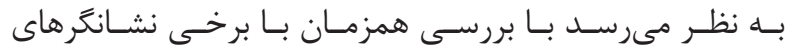

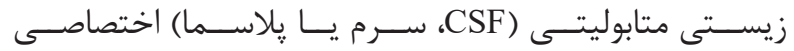

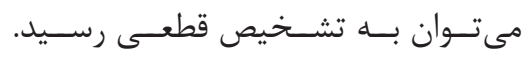

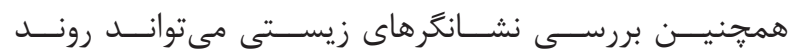

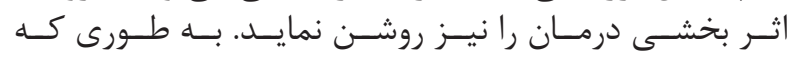

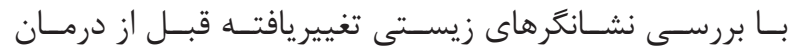

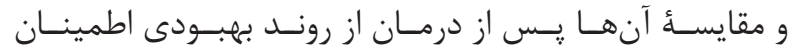

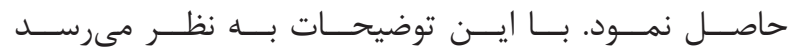

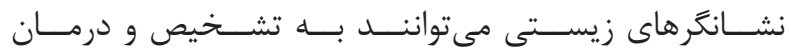

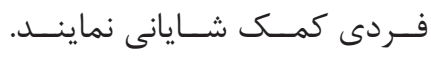

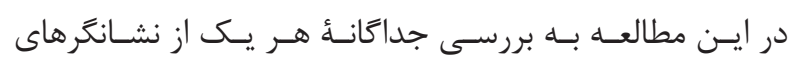

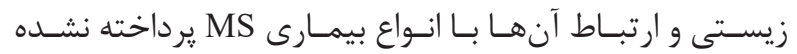

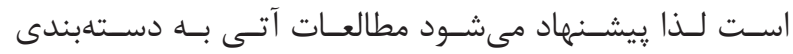

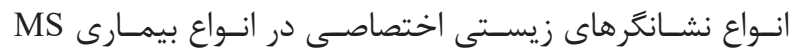

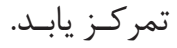

1. Weiner HL. Multiple sclerosis is an inflammatory T-cell-mediated autoimmune disease. Arch Neurol. 2004; 61(10): 1613-5.

2. Brück W. The pathology of multiple sclerosis is the result of focal inflammatory demyelination with axonal damage. J Neurol. 2005; 252(5): v3-v9.

3. Finkelsztejn A, Gabbai AA, Fragoso YD, Carrá A, Macías-Islas MA, Arcega-Revilla R, et al. Latin American algorithm for treatment of relapsing-remitting multiple sclerosis using disease-modifying agents. Arquivos De neuro-Psiquiatria. 2012; 70(10): 799-806.

4. Rodi M, Dimisianos N, de Lastic A-L, Sakellaraki P, Deraos G, Matsoukas J, et al. Regulatory cell populations in relapsing-remitting multiple sclerosis ( $\mathrm{rms}$ ) patients: effect of disease activity and treatment regimens. Int J Mol Sci. 2016; 17(9): 1398. doi: 10.3390/ijms17091398.

5. Lesage E, Apps M, Hayter A, Beckmann C, Barnes D, Langdon D, et al. Cerebellar information processing in relapsing-remitting multiple sclerosis (RRMS). Behav Neurol. 2010; 23(1-2): 39-49.

6. Hurwitz BJ. The diagnosis of multiple sclerosis and the clinical subtypes. Ann Indian Acad Neurol. 2009; 12(4): 226-30.

7. Hempel S, Graham GD, Fu N, Estrada E, Chen AY, Miake-Lye I, et al. A systematic review of the effects of modifiable risk factor interventions on the progression of multiple sclerosis. Mult Scler. 2017; 23(4): 513-24.

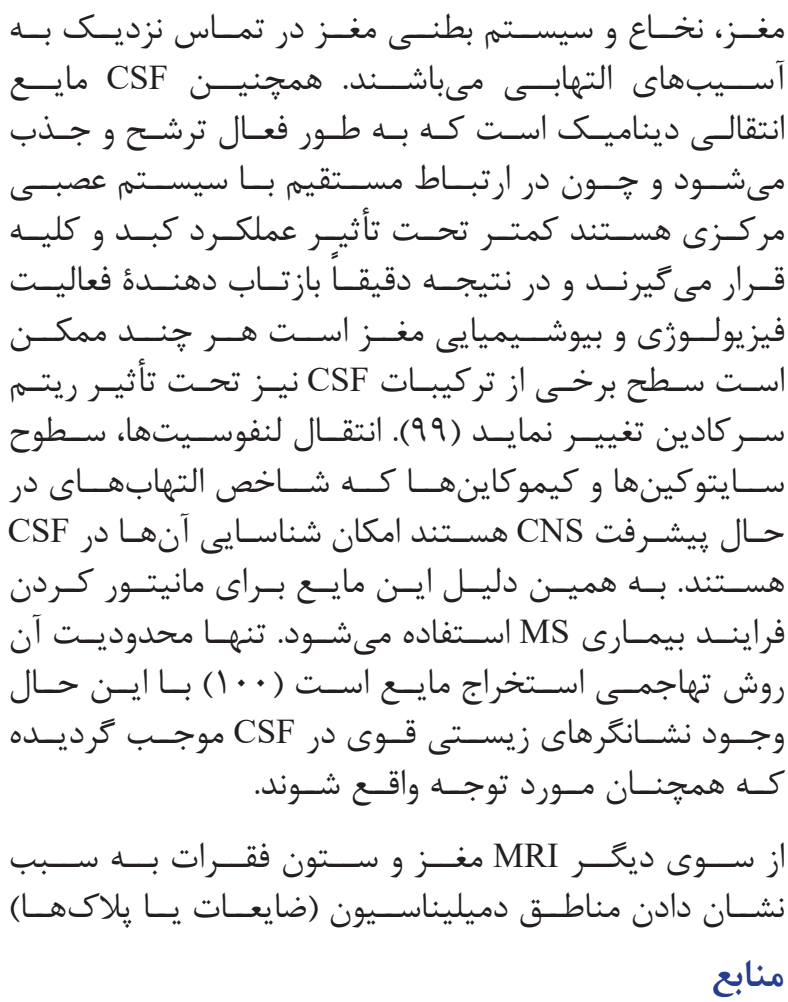

8. Brochet B, Deloire MS, Perez P, Loock T, Baschet L, Debouverie M, et al. Double-blind controlled randomized trial of cyclophosphamide versus methylprednisolone in secondary progressive multiple sclerosis. Plos One. 2017; 12(1): e0168834.

9. Murray E, Buttner E, Price B. Depression and psychosis in neurological practice. Daroff R, Fenichel G, Jankovic J, Mazziotta J Bradley's neurology in clinical practice Philadelphia. $6^{\text {th }}$ ed. PA: Elsevier/Saunders ISBN. 2012: p. 1-4377.

10. Zarei M, Chandran S, Compston A, Hodges J. Cognitive presentation of multiple sclerosis: evidence for a cortical variant. JJ Neurol Neurosurg Psychiatr. 2003; 74(7): 872-7.

11. Hauser SL, Oksenberg JR. The neurobiology of multiple sclerosis: genes, inflammation, and neurodegeneration. Neuron. 2006; 52(1): 61-76.

12. Petzold A, Gveric D, Groves M, Schmierer K, Grant $\mathrm{D}$, Chapman M, et al. Phosphorylation and compactness of neurofilaments in multiple sclerosis: indicators of axonal pathology. Exp Neurol. 2008; 213(2): 326-35.

13. Handel AE, Giovannoni G, Ebers GC, Ramagopalan $\mathrm{SV}$. Environmental factors and their timing in adultonset multiple sclerosis. Nat Rev Neurol. 2010; 6(3): 156-66.

14. Patel J, Balabanov R. Molecular mechanisms of oligodendrocyte injury in multiple sclerosis and 
experimental autoimmune encephalomyelitis. Int J Mol Sci. 2012; 13(8): 10647-59.

15. Correale J, Farez MF. The role of astrocytes in multiple sclerosis progression. Front Neurol. 2015; 6: 180 doi: 10.3389/fneur.2015.00180.

16. Constantinescu C, Gran B. The essential role of $\mathrm{T}$ cells in multiple sclerosis: a reappraisal. Biomed J. 2014; 37(2): 34-40.

17. Vogel DY, Vereyken EJ, Glim JE, Heijnen PD, Moeton M, van der Valk P, et al. Macrophages in inflammatory multiple sclerosis lesions have an intermediate activation status. J Neuroinflammation. 2013; 10(1): 35. doi: 10.1186/1742-2094-10-35.

18. Klotz L, Kuzmanov I, Hucke S, Gross CC, Posevitz V, Dreykluft A, et al. B7-H1 shapes T-cell-mediated brain endothelial cell dysfunction and regional encephalitogenicity in spontaneous CNS autoimmunity. Proc Natl Acad Sci USA. 2016; 113(41): E6182-E91.

19. W Kamphuis W, Derada Troletti C, Reijerkerk A, A Romero I, E de Vries H. The blood-brain barrier in multiple sclerosis: microRNAs as key regulators. CNS Neurol Disord Drug Targets. 2015; 14(2): 157-67.

20. Ciccarelli O, Barkhof F, Bodini B, De Stefano N, Golay X, Nicolay K, et al. Pathogenesis of multiple sclerosis: insights from molecular and metabolic imaging. Lancet Neurol. 2014; 13(8): 807-22.

21. Polman $\mathrm{CH}$, Reingold SC, Banwell B, Clanet M, Cohen JA, Filippi M, et al. Diagnostic criteria for multiple sclerosis: 2010 revisions to the McDonald criteria. Ann Neurol. 2011; 69(2): 292-302

22. Roncaroli F, Campus CC. Neuropathology of Multiple sclerosis. Adv Clin Neurosci Rehabil. 2005; 5(2): 16-22.

23. Obeidat AZ, Jacob C, Zabeti A. Concurrent dawson's fingers and area postrema lesion in a mixed neuroimmune disorder. Can J Neurol Sci. 2017; 44(4): 452-4.

24. Garcia MA, Blasco M. Confirming the MS diagnosis. Int MS J. 2007; 14(2): 58-63.

25. McDonald WI, Compston A, Edan G, Goodkin D, Hartung HP, Lublin FD, et al. Recommended diagnos tic criteria for multiple sclerosis: guidelines from the international panel on the diagnosis of multiple sclerosis. Annals of Neurology. 2001; 50(1): 121-7.
26. Aronson JK. Biomarkers and surrogate endpoints. Br J Clin Pharmacol. 2005; 59(5): 491-4.

27. Harris VK, Sadiq SA. Disease biomarkers in multiple sclerosis. Mol Diagn Ther. 2009; 13(4): 225-44

28. Storch MK, Stefferl A, Brehm U, Weissert R, Walls tröm E, Kerschensteiner M, et al. Autoimmunity to myelin oligodendrocyte glycoprotein in rats mimics the spectrum of multiple sclerosis pathology. Brain Pathol. 1998; 8(4): 681-94.

29. Xiao B-G, Linington C, Link Hd. Antibodies to myelin-oligodendrocyte glycoprotein in cerebrospinal fluid from patients with multiple sclerosis and controls. J Neuroimmunol. 1991; 31(2): 91-6.

30. Sargento-Freitas J, Batista S, Macario C, Matias F, Sousa L. Clinical predictors of an optimal response to natalizumab in multiple sclerosis. J Clin Neurosci. 2013; 20(5): 659-62.

31. Sormani MP, De Stefano N. Defining and scoring response to IFN- $\beta$ in multiple sclerosis. Nat Rev Neurol. 2013; 9(9): 504-12.

32. Berger T, Rubner P, Schautzer F, Egg R, Ulmer H, Mayringer I, et al. Antimyelin antibodies as a predictor of clinically definite multiple sclerosis after a first demyelinating event. N Engl J Med. 2003; 349(2): 139-45.

33. Brilot F, Dale RC, Selter RC, Grummel V, Reddy Kalluri S, Aslam M, et al. Antibodies to native myelin oligodendrocyte glycoprotein in children with inflammatory demyelinating central nervous system disease. Ann Neurol. 2009; 66(6): 833-42.

34. Klawiter EC, Piccio L, Lyons J-A, Mikesell R, O'connor KC, Cross AH. Elevated intrathecal myelin oligodendrocyte glycoprotein antibodies in multiple sclerosis. Arch Neurol. 2010; 67(9): 1102-8.

35. Bielekova B, Martin R. Development of biomarkers in multiple sclerosis. Brain. 2004; 127(7): 1463-78.

36. Humme S, Reisbach G, Feederle R, Delecluse H-J, Bousset K, Hammerschmidt W, et al. The EBV nuclear antigen 1 (EBNA1) enhances $\mathrm{B}$ cell immortalization several thousandfold. Proc Natl Acad Sci U S A. 2003; 100(19): 10989-94.

37. Young LS, Rickinson AB. Epstein-Barr virus: 40 years on. Nat Rev Cancer. 2004; 4(10): 757-68.

38. Levitskaya J, Coram M, Levitsky V, Imreh S. Inhibition of antigen processing by the internal repeat 


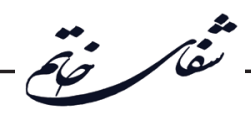

region of the Epstein-Barr virus nuclear antigen-1. Nature. 1995; 375(6533): 685-8.

39. Wang H, Munger K, Reindl M, O'reilly E, Levin L, Berger T, et al. Myelin oligodendrocyte glycoprotein antibodies and multiple sclerosis in healthy young adults. Neurology. 2008; 71(15): 1142-6.

40. Escandón-Vargas K, Zorrilla-Vaca A, Corral-Prado RH. Positive 14-3-3 and tau proteins in a sporadic Creutzfeldt-Jakob disease case and a brief perspective of prion diseases in Colombia. Biomédica. 2016; 36: 29-36.

41. Foote M, Zhou Y. 14-3-3 proteins in neurological disorders. Int J Biochem Mol Biol. 2012; 3(2): 152-64.

42. Vazifehkhah S, Karimzadeh F. Parkinson disease: from pathophysiology to the animal models. Shefaye Khatam. 2016; 4(3): 91-102.

43. Rangel-Barajas C, Coronel I, Florán B. Dopamine receptors and neurodegeneration. Aging Dis. 2015; 6(5): 349-68.

44. Haarmann AM, Jafarian M, Karimzadeh F, Gorji A. Modulatory effects of dopamine D. Basic Clin Neurosci. 2014; 5(4): 246-52.

45. Satoh J-i, Yamamura T, Arima K. The 14-3-3 protein $\varepsilon$ isoform expressed in reactive astrocytes in demyelinating lesions of multiple sclerosis binds to vimentin and glial fibrillary acidic protein in cultured human astrocytes. Am J Pathol. 2004; 165(2): 577-92.

46. Shimada T, Fournier AE, Yamagata $K$. Neuroprotective function of 14-3-3 proteins in neurodegeneration. Biomed Res Int. 2013; 2013 doi: $10.1155 / 2013 / 564534$.

47. Eliasson C, Sahlgren C, Berthold C-H, Stakeberg J, Celis JE, Betsholtz C, et al. Intermediate filament protein partnership in astrocytes. J Biol Chem. 1999; 274(34): 23996-4006.

48. Lee D-H, Steinacker P, Seubert S, Turnescu T, Melms A, Manzel A, et al. Role of glial 14-3-3 gamma protein in autoimmune demyelination. J Neuroinflammation. 2015; 12(1): 187. doi: 10.1186/s12974-015-0381-x.

49. Martínez-Yélamos A, Saiz A, Sanchez-Valle R, Casado V, Ramon J, Graus F, et al. 14-3-3 protein in the CSF as prognostic marker in early multiple sclerosis. Neurology. 2001; 57(4): 722-4.

50. Obsil T, Obsilova V. Structural basis of 14-3-3 protein functions. Semin Cell Dev Biol. 2011; 22(7): 663-72.

51. Terzi M, Birinci A, Cetinkaya E, Onar M. Cerebrospinal fluid total tau protein levels in patients with multiple sclerosis. Acta Neurol Scand. 2007; 115(5): $325-30$.

52. Kapaki E, Paraskevas GP, Michalopoulou M, Kilidireas K. Increased cerebrospinal fluid tau protein in multiple sclerosis. Eur Neurol. 2000; 43(4): 228-32.

53. Valis M, Talab R, Stourac P, Andrys C, Masopust J. Tau protein, phosphorylated tau protein and betaamyloid42 in the cerebrospinal fluid of multiple sclerosis patients. Neuro Endocrinol Lett. 2008; 29(6): 971-6.

54. Duan Y, Dong S, Gu F, Hu Y, Zhao Z. Advances in the pathogenesis of Alzheimer's disease: focusing on tau-mediated neurodegeneration. Transl Neurodegener. 2012; 1(1): 24 . doi: 10.1186/2047-9158-1-24.

55. Axelsson M, Malmeström C, Nilsson S, Haghighi S, Rosengren L, Lycke J. Glial fibrillary acidic protein: a potential biomarker for progression in multiple sclerosis. J Neurol. 2011; 258(5): 882-8.

56. Norgren N, Sundström P, Svenningsson A, Rosengren L, Stigbrand T, Gunnarsson M. Neurofilament and glial fibrillary acidic protein in multiple sclerosis. Neurology. 2004; 63(9): 1586-90.

57. Malmeström C, Haghighi S, Rosengren L, Andersen $\mathrm{O}$, Lycke J. Neurofilament light protein and glial fibrillary acidic protein as biological markers in MS. Neurology. 2003; 61(12): 1720-5.

58. Kaeser SA, Herzig MC, Coomaraswamy J, Kilger E, Selenica M-L, Winkler DT, et al. Cystatin C modulates cerebral $\beta$-amyloidosis. Nature Genet. 2007; 39(12): 1437-9.

59. Vranová HP, Sládková V, Mareš J, Hluštík P, Langová J, Kanovský P. Cystatin C as a Marker of Degeneration in Multiple Sclerosis (P03. 241). Neurology. 2013; 80(7): P03. 241-P03 .

60. Lehmann-Horn K, Kronsbein HC, Weber MS. Targeting B cells in the treatment of multiple sclerosis: recent advances and remaining challenges. Ther Adv Neurol Disord. 2013; 6(3): 161-73.

61. Disanto G, Morahan J, Barnett M, Giovannoni G, Ramagopalan S. The evidence for a role of B cells in multiple sclerosis. Neurology. 2012; 78(11): 823-32

62. Krumbholz M, Meinl E. B cells in MS and 
NMO: pathogenesis and therapy. Seminars in Immunopathology; 2014. Springer.

63. Hahne M, Renno $T$, Schroeter $M$, Irmler $M$, French L, Bornand T, et al. Activated B cells express functional Fas ligand. European Eur J Immunol. 1996; 26(3): 721-4.

64. D'souza SD, Bonetti B, Balasingam V, Cashman NR, Barker PA, Troutt AB, et al. Multiple sclerosis: Fas signaling in oligodendrocyte cell death. J Exp Med. 1996; 184(6): 2361-70.

65. Baker D, Marta M, Pryce G, Giovannoni G, Schmierer K. Memory B cells are major targets for effective immunotherapy in relapsing multiple sclerosis. EBioMedicine. 2017; 16: 41-50.

66. Ohsawa I, Takamura C, Kohsaka S. Fibulin-1 binds the amino-terminal head of $\beta$-amyloid precursor protein and modulates its physiological function. J Neurochem. 2001; 76(5): 1411-20.

67. Kanoh Y, Ohara T, Kanoh M, Akahoshi T. Serum matrix metalloproteinase-2 levels indicate blood-CSF barrier damage in patients with infectious meningitis. Inflammation. 2008; 31(2): 99-104.

68. Kouwenhoven M, Özenci V, Gomes A, Yarilin D, Giedraitis V, Press R, et al. Multiple sclerosis: elevated expression of matrix metalloproteinases in blood monocytes. J Autoimmun. 2001; 16(4): 463-70.

69. Fernandes KS, Brum DG, Sandrim VC, Guerreiro CT, Barreira AA, Tanus-Santos JE. Matrix metalloproteinase-9 genotypes and haplotypes are associated with multiple sclerosis and with the degree of disability of the disease. J Neuroimmunol. 2009; 214(1): 128-31.

70. Ottervald J, Franzén B, Nilsson K, Andersson LI, Khademi M, Eriksson B, et al. Multiple sclerosis: Identification and clinical evaluation of novel CSF biomarkers. J Proteomics. 2010; 73(6): 1117-32.

71. Mattsson N, Axelsson M, Haghighi S, Malmeström C, Wu G, Anckarsäter R, et al. Reduced cerebrospinal fluid BACE1 activity in multiple sclerosis. Mult Scler. 2009; 15(4): 448-54.

72. Willem M, Garratt AN, Novak B, Citron M, Kaufmann S, Rittger A, et al. Control of peripheral nerve myelination by the $\beta$-secretase BACE1. Science. 2006; 314(5799): 664-6.

73. Zheng P, Wang Q, Teng J, Chen J. Calumenin and fibulin-1 on tumor metastasis: Implications for pharmacology. Pharmacol Res. 2015; 99: 11-5.
74. Mandrioli J, Sola P, Bedin R, Gambini M, Merelli E. A multifactorial prognostic index in multiple sclerosis. $\mathrm{J}$ Neurol. 2008; 255(7): 1023-31.

75. Owens GP, Bennett JL, Lassmann H, O’Connor KC, Ritchie AM, Shearer A, et al. Antibodies produced by clonally expanded plasma cells in multiple sclerosis cerebrospinal fluid. Ann Neurol. 2009; 65(6): 639-49.

76. Mareš J, Herzig R, Urbanek K, Sladkova V, Opavský $\mathrm{R}$, Hluštík $\mathrm{P}$, et al. Correlation of the $\mathrm{IgG}$ index and oligoclonal bands in the CSF of patients with multiple sclerosis. Biomed Pap Med Fac Univ Palacky Olomouc Czech Repub. 2008; 152(2): 247-9.

77. Stangel M, Fredrikson S, Meinl E, Petzold A, Stüve $\mathrm{O}$, Tumani $\mathrm{H}$. The utility of cerebrospinal fluid analysis in patients with multiple sclerosis. Nat Rev Neurol. 2013; 9(5): 267-76.

78. Presslauer S, Milosavljevic D, Brücke T, Bayer P, Hübl W. Elevated levels of kappa free light chains in CSF support the diagnosis of multiple sclerosis. J Neurol. 2008; 255(10): 1508-14.

79. Presslauer S, Milosavljevic D, Huebl W, AbouleneinDjamshidian F, Krugluger W, Deisenhammer F, et al. Validation of kappa free light chains as a diagnostic biomarker in multiple sclerosis and clinically isolated syndrome: A multicenter study. Mult Scler. 2016; 22(4): 502-10.

80. Ontaneda D, Fox RJ. Progressive multiple sclerosis. Curr Opin Neurol. 2015; 28(3): 237-43.

81. Iacobaeus E, Amoudruz P, Ström M, Khademi M, Brundin L, Hillert J, et al. The expression of VEGF-A is down regulated in peripheral blood mononuclear cells of patients with secondary progressive multiple sclerosis. PLos One. 2011; 5(6): e19138.

82. Yang M, Qin Z, Zhu Y, Li Y, Qin Y, Jing Y, et al. Vitamin D-binding protein in cerebrospinal fluid is associated with multiple sclerosis progression. Molecular Neurobiology. 2013; 47(3): 946-56.

83. Perga S, Albo AG, Lis $\mathrm{K}$, Minari N, Falvo $\mathrm{S}$, Marnetto F, et al. Vitamin D binding protein isoforms and apolipoprotein $\mathrm{E}$ in cerebrospinal fluid as prognostic biomarkers of multiple sclerosis. Plos One. 2015; 10(6): e0129291.

84. Pierrot-Deseilligny C. Clinical implications of a possible role of vitamin D in multiple sclerosis. J Neurol. 2009; 256(9): 1468-79.

85. Bowling AC. Optimal health with multiple sclerosis: 


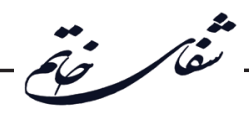

a guide to integrating lifestyle, alternative, and conventional medicine. New York: Demos Medical Publishing. 2014.

86. Stewart N, Simpson S, van der Mei I, Ponsonby A-L, Blizzard L, Dwyer T, et al. Interferon- $\beta$ and serum 25-hydroxyvitamin D interact to modulate relapse risk in MS. Neurology. 2012; 79(3): 254-60.

87. Alharbi FM. Update in vitamin D and multiple sclerosis. Neurosciences (Riyadh). 2015; 20(4): 32935 .

88. Home C. The role of vitamin $\mathrm{d}$ in multiple sclerosis pathology and treatment: answers and opportunities. International Journal of MS Care. 2015; 17(2): 1-24.

89. Munger KL, Zhang S, O’reilly E, Hernan M, Olek M, Willett W, et al. Vitamin D intake and incidence of multiple sclerosis. Neurology. 2004; 62(1): 60-5.

90. Sandberg L, Biström M, Salzer J, Vågberg M, Svenningsson A, Sundström P. Vitamin D and axonal injury in multiple sclerosis. Mult Scler. 2016; 22(8): 1027-31.

91. Jagot F, Davoust N. Is it worth considering circulating microRNAs in Multiple Sclerosis? Front Immunol. 2016; 7.doi.org/10.3389/fimmu.2016.00129.

92. Vistbakka J, Hagman S, Elovaara I. Circulating MicroRNAs as biomarkers in chronic progressive multiple sclerosis. Multiple Sclerosis Journal. Sage Publications Ltd 1 Olivers Yard, 55 City Road, London Ec1y 1sp, England. 2015.

93. Tufekci KU, Oner MG, Genc S, Genc K. MicroRNAs and multiple sclerosis. Autoimmune Diseases. 2011;
2011. doi.org/10.4061/2011/807426.

94. cd S, Hecker M, Zettl UK, Fuellen G, Taher L. Analysis of microRNA and Gene expression profiles in multiple sclerosis: integrating interaction data to uncover regulatory mechanisms. Sci Rep. 2016; 6: 1-14.

95. Quinn JF, Patel T, Wong D, Das S, Freedman JE, Laurent LC, et al. Extracellular RNAs: development as biomarkers of human disease. J Extracell Vesicles. 2015; 4(1): 27495 .

96. Ma X, Zhou J, Zhong Y, Jiang L, Mu P, Li Y, et al. Expression, regulation and function of microRNAs in multiple sclerosis. Int J Med Sci. 2014; 11(8): 810-8.

97. Tchetverikov I, Lohmander L, Verzijl N, Huizinga T, TeKoppele J, Hanemaaijer R, et al. MMP protein and activity levels in synovial fluid from patients with joint injury, inflammatory arthritis, and osteoarthritis. Ann Rheum Dis. 2005; 64(5): 694-8.

98. Perry MG, Kirwan J, Jessop D, Hunt L. Overnight variations in cortisol, interleukin 6 , tumour necrosis factor $\alpha$ and other cytokines in people with rheumatoid arthritis. Ann Rheum Dis. 2009; 68(1): 63-8.

99. Slats D, AHR Claassen J, Jan Lammers G, J Melis R, M Verbeek M, Overeem S. Association between hypocretin-1 and amyloid- $\beta 42$ cerebrospinal fluid levels in Alzheimer's disease and healthy controls. Curr Alzheimer Res. 2012; 9(10): 1119-25.

100. Tremlett H, Dai DL, Hollander Z, Kapanen A, Aziz T, Wilson-McManus JE, et al. Serum proteomics in multiple sclerosis disease progression. J Proteomics. 2015; 118: 2-11. 